\title{
Planning ahead: How recent experience with structures and words changes the scope of linguistic planning
}

\author{
Agnieszka E. Konopka \\ University of Illinois at Urbana-Champaign, Max Planck Institute for Psycholinguistics, P.O. Box 310, 6500 AH Nijmegen, The Netherlands
}

\section{A R T I C L E I N F O}

\section{Article history:}

Received 18 June 2010

revision received 18 August 2011

Available online 19 September 2011

\section{Keywords:}

Language production

Sentence planning

Incrementality

Syntactic priming

\begin{abstract}
A B S T R A C T
The scope of linguistic planning, i.e., the amount of linguistic information that speakers prepare in advance for an utterance they are about to produce, is highly variable. Distinguishing between possible sources of this variability provides a way to discriminate between production accounts that assume structurally incremental and lexically incremental sentence planning. Two picture-naming experiments evaluated changes in speakers' planning scope as a function of experience with message structure, sentence structure, and lexical items. On target trials participants produced sentences beginning with two semantically related or unrelated objects in the same complex noun phrase. To manipulate familiarity with sentence structure, target displays were preceded by prime displays that elicited the same or different sentence structures. To manipulate ease of lexical retrieval, target sentences began either with the higher-frequency or lower-frequency member of each semantic pair. The results show that repetition of sentence structure can extend speakers' scope of planning from one to two words in a complex noun phrase, as indexed by the presence of semantic interference in structurally primed sentences beginning with easily retrievable words. Changes in planning scope tied to experience with phrasal structures favor production accounts assuming structural planning in early sentence formulation.
\end{abstract}

(c) 2011 Elsevier Inc. All rights reserved.

\section{Introduction}

Speech production entails communicating a nonverbal message via a series of words produced in a specific order. Most accounts of language production assume that message planning and linguistic planning proceed incrementally (e.g., Kempen \& Hoenkamp, 1987; Levelt, 1989): by implication, in spontaneous speech, speakers know something about what they are going to say before beginning articulation, but message planning and sentence planning continue to unfold after speech onset (Bock, Irwin, Davidon, \& Levelt, 2003; Griffin, 2001; Griffin \& Bock, 2000; Lindsley, 1975; Smith \& Wheeldon, 1999). Estimates of the extent of advance planning bear on a number of debates in psycholinguistics, as the size of the increments formulated

\footnotetext{
E-mail address: Agnieszka.Konopka@mpi.nl
}

during production is an important parameter in the mapping between messages and language and in linguistic formulation itself. Minimally, a sentence increment prepared before articulation must include a message unit that can be expressed verbally (Brown-Schmidt \& Konopka, 2008; Brown-Schmidt \& Tanenhaus, 2006) and a linguistic starting point (Bock, Irwin, \& Davidson, 2004). The upper limits of these increments are more difficult to determine, in part because speakers show large variability in planning scope. ${ }^{1}$

Traditionally, one of the main concerns in production research has been to measure information flow between the different levels of the production system (e.g., Bock \&

\footnotetext{
1 The terms planning scope, advance planning, linguistic planning, sentence planning, sentence preparation, and sentence formulation are used interchangeably to refer to processes taking place before overt articulation of a given utterance chunk (Levelt, 1989).
} 
Levelt, 1994; Levelt, 1989). Planning scope refers to the amount of information that can be processed in parallel at a particular level of encoding. Thus, studies of linguistic planning attempt to assess the average breadth of planning windows by comparing production of relatively simple sentences, with easily accessible words and simple structures, to production of sentences with more words and more complex structures. In such paradigms, planning scope has been shown to vary from clausal to subphrasal. Such large variability in planning windows, i.e., in the products of various encoding processes, makes it difficult to draw specific conclusions about the extent of advance preparation during normal sentence production.

This paper takes a different approach to measuring planning scope by focusing on the problem of variability itself. In principle, planning scope can depend on prelinguistic, linguistic, and extra-linguistic factors. Extralinguistic factors include conversational constraints, such as time pressure, that might reduce the amount of planning possible before speech onset (e.g., Ferreira \& Swets, 2002,2005 ). Under conditions of little time pressure, however, planning scope is more likely to be influenced by factors tied to the mechanics of language production, i.e., the elaboration of a message and processing constraints imposed by grammatical encoding operations. In this sense, studies of planning scope offer a unique window into the products as well as the processes involved in the formulation and execution of message and sentence plans. The goal then is to identify causes of this variability within the larger theoretical context of language production.

\section{Variability in the scope of linguistic planning}

In recent experimental work, planning scope has been assessed by comparing speech onsets for a variety of sentence types (see Meyer and Lethaus (2004), for a review), including simple time expressions (Bock et al., 2003; Kuchinsky, 2010), descriptions of two-participant events (Griffin \& Bock, 2000), and descriptions of multi-picture displays (Allum \& Wheeldon, 2007, 2009; Brown-Schmidt \& Konopka, 2008; Brown-Schmidt \& Tanenhaus, 2006; Griffin, 2001; Meyer, 1996; Meyer, Sleiderink, \& Levelt, 1998; Smith \& Wheeldon, 1999). In the production of sentences consisting of simple and complex noun phrases (The kite is...vs. The kite and the dog are...), some findings suggest that planning scope may span an entire clause and others that it is limited to single phrases or lexical items.

Smith and Wheeldon (1999) proposed that speakers begin preparing all of the elements from the first clause of a sentence before speech onset and initiate rudimentary conceptual planning for a second clause. In their experiments, speakers described displays consisting of two or three moving pictures with double-clause or single-clause sentences beginning with complex noun phrases (The $A$ and the $B$ moved up and the $C$ moved down; The $A$ and the $B$ moved up) or simple noun phrases (The A moved up and the $B$ and the $C$ moved down; The A moved up). Speech onsets were longer for double-clause than single-clause sentences, and for sentences beginning with complex than simple noun phrases, suggesting that speakers completed encoding of the first phrase before speech onset (also see
Allum \& Wheeldon, 2007; Clark \& Wasow, 1998). Sentences with a longer second clause were also initiated more slowly than sentences with a short second clause, indicating that early encoding may extend beyond the first clause.

In contrast, picture-word interference studies suggest that planning scope may be limited to subclausal increments. For example, when producing sentences like The arrow is next to the bag, speakers experience interference from auditory distractors that are semantically related to either object, implying that linguistic encoding is completed for both lemmas before speech onset (Meyer, 1996; also see Wagner, Jescheniak, \& Schriefers, 2010). Importantly, Meyer (1996) noted numerically greater interference from distractors related to the first than the second noun, possibly indicating incremental encoding of these nouns in the order of mention. Smith and Wheeldon (2004) observed a similar, but much stronger graded effect: speakers took longer to initiate sentences like The saw and the axe moved down than The saw moved below the axe, showing a higher likelihood of parallel activation of nouns from the same phrase than from different phrases.

Finally, investigations of parallel object processing within one phrase also yielded mixed results. First, Meyer (1996) observed a comparable amount of interference from distractors related to either noun in phrases like the arrow and the bag (but see Meyer, 1997). More recently, Allum and Wheeldon (2009) showed that processing of the sentence-initial noun in such phrases is more thorough than processing of the second noun. On the other hand, Griffin (2001) observed that speech onsets for longer sentences like The clock and the television are above the needle vary with the codability and frequency of the first noun alone and that retrieval of the second object name awaits completion of phonological encoding of the first object (also see Bonin, Malardier, Meot, \& Fayol, 2006; Griffin \& Spieler, 2006; Meyer, Roelofs, \& Levelt, 2003; Meyer et al., 1998). This points to a very narrow, subphrasal scope of planning in a radically incremental linguistic system.

In general, therefore, there is little consensus as to the size of average planning windows even during the encoding of short sentences, which calls into question the assumption that planning consistently proceeds in increments of a particular size. The most parsimonious conclusion is that production can be incremental to different degrees in different contexts. If so, a more informative approach may be to examine when and why speakers engage in more extensive or more limited planning.

Some accounts of linguistic planning attribute this variability to the larger production context and to speaker control. Speakers are normally subject to two pressures: they may want to start speaking as early as possible to begin communicating quickly, or they may prefer to maximize fluency by planning their utterances more extensively before speaking (Levelt \& Meyer, 2000; Martin, Crowther, Knight, Tamborello, \& Yang, 2010; also see Clark \& Wasow, 1998; Griffin, 2003). Benefits of the first choice come at the expense of broader planning of both messages and sentences, which can increase disfluency rates (Clark \& 
Clark, 1977; Clark \& Wasow, 1998; Fraundorf \& Watson, 2008) and certain types of speech errors (Dell, Burger, \& Svec, 1997). Benefits of the second choice come at the expense of communication speed and require buffering more information in working memory. While it is desirable to maintain both fast and fluent production (Clark, 2002), adult speakers often strategically choose to begin communicating quickly and plan subsequent sentence chunks during production itself. Such adjustments in planning scope can quickly be made in response to external pressures, such as when responding before a deadline (Ferreira \& Swets, 2002, 2005) or under conditions of cognitive load (Wagner et al., 2010), but may also occur during the course of a single experimental session as speakers develop strategies (implicitly or explicitly) to perform the task efficiently (also see Schriefers \& Teruel, 1999). Finally, additional variability in planning scope may also come from individual differences in speakers' working memory spans (Swets, Jacovina, \& Gerrig, 2008).

An alternative to seeing planning scope as a parameter under speaker control is to consider variability in planning windows from the perspective of basic production mechanisms. If planning scope were found to vary systematically across speakers and situations, then at least some of the variability in planning windows may be re-interpreted as a natural consequence of the coordination of processes at the interface of thinking and speaking. It is difficult to a priori predict the breadth of planning windows for specific sentence types, but it is possible to gauge what type of information consistently facilitates or constrains online planning. Candidates for factors influencing planning scope include key processes in grammatical encoding (lexical and structural processing), so comparing their contributions to sentence planning provides a test of both the products (the breadth of planning windows) and processes (grammatical encoding operations) involved in normal sentence formulation.

\section{Incrementality in sentence planning}

Questions about the time course of linguistic planning are relevant for debates about lexical-structural integration in production. Sentence formulation requires that speakers integrate information about the protagonists of an event and about the relationships between them, i.e., that they coordinate word retrieval with the construction of suitable sentence frames. Naturally, words must be inserted into the right slots within a structural frame, and the frame must have the right number of slots to accommodate these words. This relationship between lexical and structural processes makes identifying the independent contributions of words and structures to sentence formulation a notoriously difficult problem (Bock, 1987). Current proposals of how lexical-structural integration occurs address this problem by assuming that one of these processes may take precedence over the other and guide early sentence planning: words may play a role in selecting the necessary structural configurations, or structural processes may control the timing of lexical retrieval, with words becoming active when a structural frame calls for them.
Accordingly, Bock et al. (2003, 2004) propose that sentence planning can be either lexically or structurally incremental. Lexical incrementality plays out as the serial encoding of one message element at a time, with hierarchical relationships between these elements established later on the basis of the structural privileges of retrieved words. For example, a speaker might retrieve the word corresponding to one message element at the outset of sentence formulation and continue building the sentence word by word from that point (Gleitman, January, Nappa, \& Trueswell, 2007). This sequence of operations is intuitively appealing because the observable outcome of linguistic encoding is the articulation of a series of words, and is compatible with functional accounts of grammatical encoding that give lexical items a leading role in the acquisition and generation of sentence structure (Bates \& MacWhinney, 1982; Bock, 1982; Tomasello, 2000; also see Garnsey, Pearlmutter, Myers, \& Lotocky, 1997).

In contrast, structural incrementality posits that sentence formulation begins with the generation of a simple but broad sentence plan that captures the relationships between various message elements (Bock et al., 2004; Kuchinsky \& Bock, 2010). Support for structural incrementality comes from work showing longer planning times for the first content word than later words in a sentence (Bock et al., 2003; Griffin \& Bock, 2000): the additional time spent on planning the first sentence increment is taken to reflect the generation of a sentence framework that includes a possible sentential starting point as well as a possible continuation of the utterance from that point. In other words, sentence formulation need not begin with the retrieval of a word triggering the construction of a phrasal frame; rather, a frame may be in place before lexical retrieval begins and may specify the order in which words are to be retrieved. This account is compatible with abstract structural accounts of grammatical encoding that emphasize the relative self-sufficiency of structural processes in sentence production, on the assumption that the deployment of structure-building procedures is not contingent on lexical retrieval (Bock, 1990; Bock et al., 2003, 2004; Christianson \& Ferreira, 2005; Dell, 1986; Ferreira \& Dell, 2000; Fisher, 2002; Konopka \& Bock, 2009; see Chang, Dell, \& Bock (2006), for a model).

Of course, since the production system is flexible, control of sentence formulation can oscillate between lexical and structural processes (see Ferreira, 1996) and sentence structure may be derived both from a lexical and a structural source (Pickering \& Ferreira, 2008). The specific balance of lexical and structural contributions to sentence formulation can shift, for example, as speakers try to meet different communicative goals or adapt to the pressures of different communicative contexts. Although the implications of this debate are not often brought to bear on the problem of variability in planning scope, these shifts may go hand-in-hand with variability in planning windows (Ferreira \& Swets, 2002; Wagner et al., 2010): the extent of advance planning may follow from the speaker's planning strategies, and the sequence of encoding operations for any given sentence may fall somewhere on the continuum between lexical and structural incrementality, being more compatible with either at different times. 
The critical difference between the two accounts of incremental planning concerns the order of lexical and structural operations in early sentence formulation: word retrieval can proceed serially, with little need for extensive anticipatory activation of upcoming message elements, but structural planning requires that speakers know something of the relationships between different message elements or, at a minimum, know something about the role of the current sentence increment in the larger sentence context. As such, the two accounts make different predictions about factors that should modulate planning scope. Taking this approach to linguistic planning requires identifying the conditions under which speakers do and do not engage in extensive preparation, and thereby revisits this question in the context of basic production mechanisms (Bock, 1987; Bock \& Levelt, 1994; Levelt, 1989).

Lexical control of sentence formulation, or lexical incrementality, predicts that when encoding a sentence like The axe and the saw..., the speaker will retrieve the word axe without necessarily knowing that it is the first noun in a complex noun phrase (the only increment planned up to this point is a single noun). When encoding the word saw, the speaker will begin building a structure that expresses the relationship between the two objects. So, if planning begins with word retrieval, the frequency of the first word should determine when the second word is retrieved and thus when a complex noun phrase is built. If the word axe can be retrieved quickly, the speaker may begin retrieving the second word in parallel with the first, resulting in phrasal planning. If the word axe is not readily accessible, the speaker may begin retrieving the second word only after completing phonological encoding of the first, resulting in a subphrasal scope of planning. These predictions are similar to those of Griffin (2003), where speakers were able to prepare two words in parallel when the first word was short (e.g., wig carrot) but not when it was long (e.g., windmill carrot). In both cases, planning is constrained by a non-hierarchical factor.

In this scenario, the ease of building a phrasal structure should play a secondary role in early sentence planning. If speakers can only add increments of linguistic information to a developing sentence as quickly as words can be retrieved, then planning scope should be determined by the complexity of each increment alone and not by its role in the developing utterance. So, in sentences where the frequency of the first word allows parallel object processing, the ease of building this phrase will not influence the relative timing of retrieving the two object names. Similarly, in sentences where the frequency of the first word precludes parallel processing of a second object, the ease of structure building structure should not change the fact that speakers will generate the first single-noun increment (The axe...) and the second single-noun increment (...and the saw) in separate time windows, although they may be able to integrate the second increment into the developing utterance more quickly when the structure is easy to generate.

In contrast, in a structurally incremental system, the message must first generate a rudimentary or partial structural scheme that provides information about dependencies between at least some of the message elements and a possible order for them to appear in a sentence (Bock et al., 2003, 2004). Apprehension of the overall structure of a message occurs rapidly after stimulus onset (Griffin \& Bock, 2000) and allows speakers to begin mapping this structure onto a linguistic structure. Under the assumption that structural assembly precedes lexical retrieval, early sentence planning should depend primarily on processes responsible for word order. Speakers should engage in more extensive planning when they are able to map the message onto a linguistic structure more easily; conversely, when the message-to-structure mapping is more difficult, they may generate chunks of sentence structure that are as small as simple noun phrases. Importantly, in both cases, the generation of each small sentence increment begins with structure-building, followed by word retrieval.

Naturally, measurement of structural planning is only possible when speakers actually speak, so structural processes inevitably interact with lexical retrieval. The retrieval and insertion of words into slots in a structural plan is a sequential, time-consuming process (Griffin \& Bock, 2000), so lexical retrieval creates a production bottleneck that has immediate consequences for performance in any picturenaming task: retrieval costs cannot be factored out from production measures like speech onsets, so lexical frequency inevitably imposes a limit on the observability of extended sentence planning. When a sentence structure is easy to generate and the sentence-initial word can be retrieved quickly, speakers may begin encoding the second content word in close temporal succession to the first, resulting in phrasal planning. However, when retrieval of the sentence-initial word is more difficult, the speaker must delay retrieval of any subsequent words, whether a larger phrasal frame has already been generated or not, providing little evidence for the availability of a broader structural plan. Thus, since lexical frequency modulates the extent to which a structural plan can be executed online, the observable result is a scope of planning that differs in sentences beginning with accessible and less accessible words.

To date, numerous studies have examined lexical constraints on planning scope. For example, Meyer, Ouellet, and Haecker (2008; also Morgan \& Meyer, 2005) asked speakers to name object pairs in a paradigm where the second, extrafoveally viewed object (the interloper) was replaced by a different one (the target object) during a speaker's saccade to this object location. Gaze durations to the second object were shorter when the interloper was a homophone of the target object, suggesting early processing of the second object during fixations to the first object. The effect was larger when the first object was easy to name. In a different set of experiments, speakers also spent more time looking at the first object if the name of the second object was easy to retrieve, confirming that ease of naming modulates the extent of parallel object processing (Malpass \& Meyer, 2010; see Griffin, 2003).

However, it is unclear whether these effects would hold during production of full phrases: trial structure is repetitive in many object-naming studies, and speakers either do not generate syntactic structures or produce the same structure on every trial (Griffin, 2001). Repetition of either trial structure or sentence structure can place the same 
demands on production as sequential naming tasks where the most efficient way to plan a response is to encode words one by one (see Martin et al., 2010). Since this leaves the ease of retrieving the first noun as the primary factor distinguishing one item from another, the results cannot be unambiguously brought to bear on questions regarding the contrast between lexical and structural incrementality. Thus the current experiments aimed to distinguish between a lexical and a structural bottleneck in planning with a combined manipulation of the ease of word retrieval and structure building. Lexical and structural incrementality make different assumptions about the mechanics of sentence planning, so these manipulations were expected to show how planning scope might change when lexical and structural constraints are strong and when these constraints are relaxed.

\section{Current experiments}

Production involves resource-consuming and timeconsuming encoding processes (Ayora, Janssen, Dell'Acqua, \& Alario, 2009; Ferreira \& Pashler, 2002; Kuiper, 1996; Roelofs, 2008; Smith \& Wheeldon, 1999), so both lexical retrieval and structure building can exhibit large variability in encoding fluency. For example, speakers retrieve words more quickly for concepts that are contextually expected (Griffin \& Bock, 1998; Ferreira \& Pashler, 2002), more accessible (Bock \& Warren, 1985; Prat-Sala \& Branigan, 2000) or easier to code linguistically (Griffin, 2001). There are also clear cumulative frequency effects, with higher-frequency words retrieved more quickly than lower-frequency words (Griffin, 2001; Meyer et al., 1998). Recent exposure to lexical items, such as previewing pictures (Allum \& Wheeldon, 2009; Smith \& Wheeldon, 1999), seeing pictures repeatedly (Van Der Meulen, Meyer, \& Levelt, 2001), or repeatedly processing the words themselves (repetition priming; Wheeldon \& Monsell, 1992) also temporarily reduces retrieval times.

Analogous to word retrieval, structural processes are also sensitive to recent and cumulative experience. One of the strongest demonstrations of this sensitivity is the unintentional repetition of structure across sentences (see Pickering and Ferreira (2008), for a review), which has been shown with a variety of structures (Bock, 1986; Cleland \& Pickering, 2003; Gries, 2005; Konopka \& Bock, 2009; Pickering \& Branigan, 1998; Scheepers, 2003; Szmrecsanyi, 2004), tasks (Bock, 1986; Branigan, Pickering, \& Cleland, 1999, 2000; Hartsuiker, Bernolet, Schoonbaert, Speybroeck, \& Vanderelst, 2008; Hartsuiker \& Westenberg, 2000; Potter \& Lombardi, 1998; Scheepers, 2003), time intervals (Bock \& Griffin, 2000; Hartsuiker et al., 2008; Kaschak \& Borregine, 2008), and languages (Hartsuiker, Pickering, \& Veltkamp, 2004; Loebell \& Bock, 2003). Proposed functions and consequences of structural repetition include learning of structure-building procedures, faster deployment of these procedures, and speaker alignment in conversation (Ferreira \& Bock, 2006).

A link between moment-to-moment fluctuations in formulation fluency and sentence planning is yet to be established. The hypothesis evaluated here is that if familiarity with words and structures has short-term and long-term benefits for production, speakers may also prepare more or less information for an upcoming utterance as these processes become more or less efficient. So, in the current experiments, speakers described multi-picture displays with a variety of structures. On prime and target trials, speakers saw three pictures arranged to elicit sentences consisting of simple and complex noun phrases. Target trials included semantically related or unrelated pictures to be named in a complex noun phrase (e.g., The axe and the saw are above the cup), and the magnitude of semantic interference served as the primary index of temporal overlap in the retrieval of the first two nouns. To manipulate ease of lexical retrieval, sentences began either with the higher-frequency or lower-frequency semantic pairmate (The axe and the saw... vs. The saw and the axe...). To manipulate ease of structural processing, target displays were preceded by primes eliciting either the same or different structures, so that target sentences were structurally primed or unprimed. Finally, to minimize strategic selection and use of similar-sized planning windows through the experimental session, prime-target pairs were separated by three filler trials that made trial structure less predictable. Thus throughout the experiment speakers named objects that varied considerably in lexical frequency and used a variety of structures: Setting aside any extra-linguistic or task-specific pressures on sentence planning, variability in planning scope should be related to encoding difficulty.

In general, planning proceeds in subphrasal windows when encoding difficulty increases: e.g., planning windows are smaller when sentences begin with a conceptually complex than conceptually simple phrase (The blue frog. . . vs. The frog. ..), and when speakers must decide what utterance form to produce on each trial based on experimentspecific criteria (Wagner et al., 2010). So, if speakers were to primarily encode their sentences one object at a time in the current experiments, they should experience no semantic interference on target trials and speech latencies should depend on the frequency of the first word. Interference should only be obtained if the experimental manipulations promote phrasal planning on target trials by reducing processing effort. The orthogonal manipulation of lexical retrieval and structure building difficulty provides a test of whether shifts in planning scope occur by relaxing lexical or structural constraints.

If sentence planning begins with word retrieval, consistent with lexical incrementality, planning scope should depend on the frequency of the first object name. When this object is easy to name, speakers may begin processing the second object in parallel and thus experience semantic interference. Repetition of structure may reduce onsets in these sentences (Smith \& Wheeldon, 2001), but should not change the magnitude of semantic interference in primed and unprimed sentences. If the name of the first object is more difficult to retrieve, speakers might allocate most of their resources to this object and begin processing the second object as late as during articulation of the first noun. Again, this pattern should also be similar in structurally primed and unprimed sentences.

In contrast, in a structurally incremental system, planning scope should differ in primed and unprimed 
sentences. Speakers should employ a narrow, subphrasal scope of planning, initiating retrieval only for the first word (The axe...), when the structure mapping this message onto language had not been recently used. This leaves lexical frequency as the only determinant of speech latencies. In structurally primed sentences, on the other hand, reductions in processing costs associated with constructing a phrasal frame may allow for earlier retrieval of the second word than is computationally possible when speakers generate a structure anew. In light of work suggesting a strong lexical bottleneck on production, this is the predicted pattern for sentences beginning with high-frequency words: when a lower-frequency object is to be named first, any benefits of the early generation of a structural frame on sentences planning may be masked by the difficulty of lexical retrieval.

It should be noted that while these predictions are based on the finding that planning scope is generally narrow when processing is more difficult and wider when processing is easier, in theory, the reverse may also be true (Wagner et al., 2010). Planning scope may be narrow when encoding easier sentences because smaller increments can be processed quickly and efficiently, possibly to free up working memory resources before encoding the next increment. Planning scope may be wider when encoding harder sentences because speakers may prefer to encode larger units of linguistic material before speech onset to avoid speaking and planning at the same time. These choices may be more strategic than process-based, but both predictions are reasonable in a flexible system where planning patterns depend on a number of variables. The combination of these variables can create a continuum of planning windows, influenced by different variables at different ends of this continuum. Although planning scope tends to be narrow when processing is difficult (processing difficulty is an experiment-specific parameter), it is of course possible that as difficulty increases further, speakers may start encoding sentences in wider planning windows for reasons beyond the mechanistic explanations of lexical and structural incrementality. In the context of the current study, this hypothesis can be partially verified in conditions where both lexical retrieval and structural assembly are time-consuming.

The first experiment tested for changes in planning scope with objects that were either easy or hard to name and structures that were easier or harder to build due to a within-experiment manipulation of structural accessibility. To verify the claim that in a structurally incremental system, lexical frequency produces a bottleneck in production but not in planning, Experiment 2 tested whether recent changes in lexical availability produce a pattern analogous to that seen with differences in cumulative lexical frequencies. Comparable effects of lexical frequency and structural priming on phrase planning were expected in both experiments.

\section{Experiment 1}

The goal of the first experiment was to establish whether lexical frequency and structural availability modulate planning scope in complex noun phrases. Participants produced sentences describing the location of objects with high- and low-frequency names in pictorial displays with variety of syntactic structures. Target nouns in sentence-initial complex noun phrases were semantically related or unrelated and the phrasal structure of target sentences was structurally primed or unprimed. It was expected that the ease of lexical retrieval and structure building would facilitate production in all sentences (i.e., would lower onsets in all sentences with easier words and repeated structures), but would have different consequences for sentence planning.

If the ease of lexical retrieval affects planning scope, sentences beginning with semantically related, highfrequency words should have longer onsets, indicating phrasal planning, than sentences with low-frequency words, where the difficulty of lexical retrieval should preclude phrasal planning. Repetition of structure should reduce speech onsets in both cases. On the other hand, if ease of structural formulation influences early sentence planning, onsets for structurally unprimed sentences beginning with related and unrelated words should not differ, consistent with speakers planning utterances in subphrasal increments, but repetition of structure should produce a shift towards phrasal planning: primed sentences with related words should be produced more slowly than primed sentences with unrelated words, as the costs of competition between related nouns should offset any reductions in onsets caused by structural priming.

\section{Method}

\section{Participants}

One hundred and twenty-eight undergraduates from the University of Illinois participated in exchange for course credit. All were native speakers of English. Twenty were replaced because they failed to produce a minimum of $60 \%$ scorable responses.

\section{Materials and design}

Black and white pictures were selected from the Snodgrass and Vaderwart (1980) collection, the International Picture-Naming Project (Szekely et al., 2004), and from Microsoft clipart. The critical stimuli were 32 pairs of objects from the same taxonomic category (e.g., axe and saw). Each word was matched with a same-frequency, same-length, semantically unrelated control. Semantic similarity and dissimilarity between the target objects and their controls was confirmed with LSA ratings (available at http://lsa.colorado.edu). Frequency ratings were

\section{Table 1}

Mean frequencies of high-frequency (HF) and low-frequency (LF) target words and their controls (with standard errors) in both experiments.

\begin{tabular}{llr}
\hline & Set 1 & Set 2 \\
\hline HF target & $70(20)$ & $70(20)$ \\
HF control & $75(24)$ & $66(18)$ \\
LF target & $22(5)$ & $22(5)$ \\
LF control & $23(6)$ & $22(5)$ \\
\hline
\end{tabular}


(a) high-frequency word first

spatially similar prime

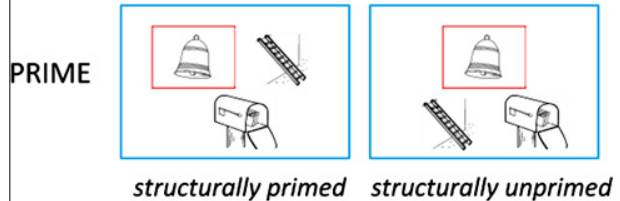

TARGET

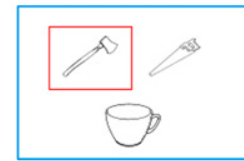

(b) low-frequency word first

spatially similar prime

PRIME
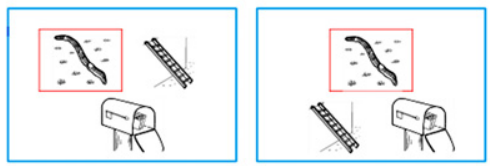

structurally primed structurally unprimed

TARGET

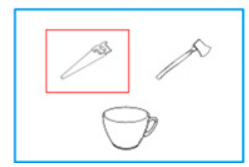

spatially different prime
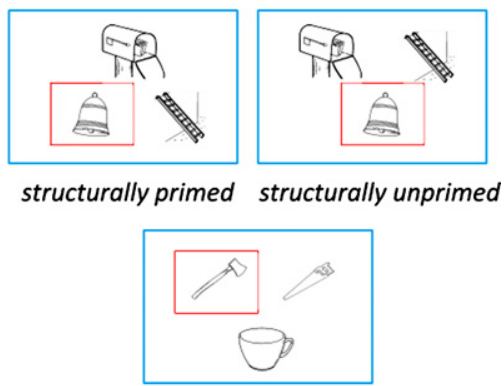

spatially different prime

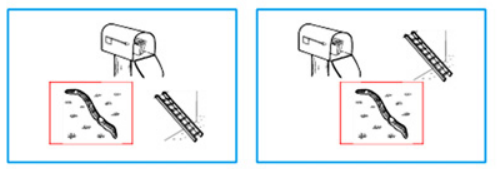

structurally primed structurally unprimed

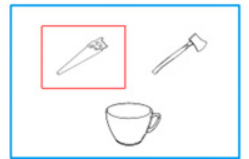

Fig. 1. Example of displays used in Experiment 1 for prime and target trials beginning with (a) high-frequency words and (b) low-frequency words in the semantically related condition. On primed trials, the arrangement of pictures on prime displays elicited complex-simple sentences; on unprimed trials, it elicited simple-complex sentences. In Experiment 2, all target sentences began with low-frequency words.

obtained from Kucera and Francis (1982) (see Appendix A and Table 1).

Two versions of experimental items were constructed with each semantic pair to minimize any idiosyncratic effects of word pairings. Each item consisted of a set of four words: two semantically related words (target words) and their two semantically unrelated controls. The controls were different in the two versions of each item set. For example, bell served as the control for axe in the first version of this item, and mouse served as the control in the second version; worm served as the control for saw in the first version, and knot served as the control in the second version of this item (two of the control words for lowfrequency target objects did not meet this criterion and are excluded from all tables and analyses). All analyses combined results from both versions of each item.

Target displays contained three objects to be named in a sentence beginning with a complex noun phrase, e.g., The axe and the saw are above/below the cup. In each display, one of the objects appeared in a red box to designate the starting point of the utterance. The object selected to appear in sentence-final position was unrelated to the target words and control words. A different object was used for items in the first and second versions of each item set.

Prime displays also contained three objects. Because of a small number of available pictures with similarfrequency names, the first picture in the prime displays was the frequency-matched control for the first picture appearing in the target sentence. ${ }^{2}$ The second and third words were selected randomly. None of these pictures were semantically or phonologically related to one another or to pictures shown on target trials.

The experiment had a three-factor within-participant and within-item factorial design (see Fig. 1), crossing the factors of semantic relatedness of the target words (semantically related vs. unrelated words), frequency of the first word (higher- vs. lower-frequency pairmate), and structural priming (primed vs. unprimed complex noun phrases). The items were arranged in eight lists, with each item appearing in a different condition in each list. Within lists, half of the target displays contained two semantically related pictures (the axe and the saw), and half contained one of the objects from the semantic pair followed by the frequency-matched control for the second object in that pair (the axe and the worm). To manipulate ease of lexical retrieval, the order of objects was counterbalanced, with sentences beginning with the higherfrequency pairmate (The axe and the saw...) half the time and the lower-frequency pairmate (The saw and the

\footnotetext{
2 The second experiment used the same set of three pictures in all prime sentences and the results were not different from those of Experiment 1, so it is unlikely that the frequency of the first word in the prime reliably influenced planning patterns in target sentences in this study.
} 
axe...) the other half. To manipulate familiarity with structure, the arrangement of objects in the prime display elicited utterances beginning with a complex noun phrase followed by a simple noun phrase (e.g., The bell and the ladder are above/below the mailbox) half the time, and utterances beginning with a simple noun phrase the other half (e.g., The bell is above/below the ladder and the mailbox). Each participant saw four items in each cell of the design, and each item was seen by 16 participants in each cell of the design.

The design also included a fourth factor to counterbalance familiarity with message structure, operationalized as visual (or perceptual) similarity in the locations of pictures in prime and target displays: the spatial arrangement of pictures was similar in prime and target displays half the time and different the other half. In similar displays, the sentence-initial object appeared in the same row of the display on prime and target trials; in spatially dissimilar displays, the sentence-initial object appeared in different rows on prime and target trials (Fig. 1). This factor was also manipulated within-participants and within-items, so eight additional lists were created to show each item in a spatially similar and spatially dissimilar condition on different lists. The location of the picture shown in the red box, indicating the starting point for each sentence, was also counterbalanced: in half of the displays on prime and target trials, the sentence-initial object appeared in the top row, and in the other half it appeared in the bottom row. This manipulation was used in both experiments, but discussion of spatial effects on planning is postponed to the 'General discussion'.

Within lists, items were arranged so that no two items from the same condition were presented back to back. There were no more than two consecutive prime-target pairs with either semantically related or semantically unrelated words, and no more than three consecutive primetarget pairs beginning with a high- or low-frequency word. Prime-target pairs alternated between structurally primed and unprimed conditions. Each prime-target pair also had a spatially different arrangement of pictures from adjacent pairs.

Prime-target pairs were separated by three fillers. To reduce the predictability of displays and structures from trial to trial, the fillers consisted of one-, two-, and threepicture displays that elicited five sentence types different from those used on prime and target trials, but also consisting of simple and complex noun phrases. Eighty-eight of the pictures on filler trials were presented once; the remaining 44 pictures were presented twice on different filler trials. Altogether, the experimental session consisted of 160 trials.

\section{Norming}

Since semantically-related words are frequently encountered in a specific order in conjoined noun phrases (e.g., king and queen, but not queen and king; Cooper \& Ross, 1975), it was important to rule out differences in the acceptability of the two word orders as an additional influence on speech latencies. Seventy-one new participants received a list of complex noun phrases with semantically related words (the axe and the saw; the saw and the axe) and the same words paired with their controls (the axe and the worm; the saw and the bell), and indicated how acceptable each phrase sounded to them on a 1-5 Likert scale ( $1=$ not natural, $5=$ very natural). Both orders of semantically related words were judged as sounding very natural, although semantic pairs beginning with high-frequency words received slightly higher ratings $(M=4.32)$ than pairs beginning with low-frequency words $(M=4.16)$, $t(31)=3.18$. The ratings for the pairings of high- and low-frequency target words with their controls $(M=2.20$ and 2.03 respectively) did not differ from one another, $t(31)=1.38$

\section{Procedure}

Participants were tested individually on a Macintosh Quadra 650 running PsyScope software (Cohen, MacWhinney, Flatt, \& Provost, 1993). They were asked to describe the location of pictures on the screen, starting with the object in a red box. The experimenter showed participants 13 displays with all the possible picture arrangements they would see in the experiment. Each display included a printed sentence below the pictures which the experimenter read to participants as she explained the task. Participants were instructed to use only these structures and completed a practice phase consisting of 13 trials eliciting the structures they had just seen. The experiment was self-paced, but participants were asked to respond as quickly as possible while maintaining fluency. Responses were recorded through a head-worn Shure microphone and transcribed later from audiofiles.

\section{Scoring and analyses}

Target sentences were scored only if participants produced both the prime and target sentences with the intended structures and used the expected words on target trials. Targets sentences produced with incorrect structures (e.g., The axe is next to the saw and below the cup), incorrect word order, or preceded by prime trials where speakers used the wrong structure were rejected $(6 \%$ of all trials). Word substitutions in target sentences were not accepted unless the new word was of the same length and similar frequency as the intended noun (e.g., bookbag for backpack). Sentences where speakers referred to a picture with the name of another category member (e.g., deer for moose), a repeated word (e.g., the hat and the other hat), a superordinate term (e.g., bug for fly), a modified noun phrase (e.g., the bird's nest), an unrelated word, or trials with technical problems were not accepted $(17 \%$ of all trials). Sentences with verbal disfluencies produced after speech onset and before the second noun were also rejected ( $1 \%$ of all trials).

First article and first noun onsets were recorded for each remaining target trial, eliminating sentence-initial disfluencies like $u$ h's, uhm's, lip smacks, or other noises. Since the experimental hypotheses concerned the production of words within sentences, all analyses were conducted on first article onsets. Data trimming was carried out in two steps. Speakers often paused briefly between production of the first article and first noun, and the distribution of onset differences between these two words 
Table 2

Mean onset differences (with standard errors) between the first article and first noun across conditions in both experiments.

\begin{tabular}{|c|c|c|c|c|c|}
\hline \multirow{3}{*}{ Frequency of first noun } & \multirow{3}{*}{ Structure } & \multicolumn{2}{|c|}{ Experiment 1} & \multicolumn{2}{|c|}{ Experiment 2} \\
\hline & & \multicolumn{2}{|c|}{ Semantic relatedness } & \multicolumn{2}{|c|}{ Semantic relatedness } \\
\hline & & Neutral & Semantic & Neutral & Semantic \\
\hline \multirow[t]{2}{*}{ High-frequency } & Primed & $154(7)$ & $178(12)$ & $138(5)$ & $132(6)$ \\
\hline & Unprimed & $150(6)$ & $170(10)$ & $145(7)$ & $138(9)$ \\
\hline \multirow[t]{2}{*}{ Low-frequency } & Primed & $147(6)$ & $156(10)$ & $146(10)$ & $159(11)$ \\
\hline & Unprimed & $144(7)$ & $157(8)$ & $144(7)$ & $160(13)$ \\
\hline
\end{tabular}

Table 3

Number (and proportions) of excluded trials across conditions in both experiments.

\begin{tabular}{|c|c|c|c|c|c|}
\hline \multirow{3}{*}{ Frequency of first noun } & \multirow{3}{*}{ Structure } & \multicolumn{2}{|c|}{ Experiment 1} & \multicolumn{2}{|c|}{ Experiment 2} \\
\hline & & \multicolumn{2}{|c|}{ Semantic relatedness } & \multicolumn{2}{|c|}{ Semantic relatedness } \\
\hline & & Neutral & Semantic & Neutral & Semantic \\
\hline \multirow[t]{2}{*}{ High-frequency } & Primed & $148(.04)$ & $137(.03)$ & $90(.03)$ & $91(.03)$ \\
\hline & Unprimed & $160(.04)$ & $180(.04)$ & $112(.04)$ & $107(.03)$ \\
\hline \multirow[t]{2}{*}{ Low-frequency } & Primed & $125(.03)$ & $143(.03)$ & $98(.03)$ & $108(.04)$ \\
\hline & Unprimed & $152(.04)$ & $174(.04)$ & $107(.03)$ & $134(.04)$ \\
\hline
\end{tabular}

showed that pause length varied by condition (Table 2), with longer pauses and greater variability in pause durations in sentences with related words. To take these differences into account, each data point was compared against a condition-appropriate cutoff to exclude trials with long pauses and long onsets as follows. First, to ensure that only fluent sentences were included in the analyses, sentences with onset differences between the first article and the first noun longer than two standard deviations away from the condition mean were excluded. Second, all remaining sentences in which sentence onsets were longer than two standard deviations of the corresponding condition mean were also excluded from the analyses, leaving 2877 trials in the final dataset (70.2\% of all trials). The distribution of errors across conditions is shown in Table 3.

The data were submitted to three-factor analyses of variance, with the crossed factors of semantic relatedness, lexical frequency, and structural priming, followed by planned comparisons (Rosenthal \& Rosnow, 1985). Figures show participant means, with $95 \%$ confidence intervals for pairwise planned comparisons. All effects are considered to be reliable at the $\alpha<.05$ level, unless specified otherwise. Because of a large number of empty cells, these analyses were followed by mixed-effects modeling, using participants and items as random effects (Baayen, Davidson, \& Bates, 2008). Results are listed in Appendix B. Additional analyses for both experiments, including effects of spatial similarity between primes and targets as well as analyses of speaker characteristics are presented in the 'General discussion'.

Results

As expected, the ease of retrieving the first noun and building structures facilitated linguistic encoding: speech onsets were shorter in sentences beginning with higher- frequency words than lower-frequency words in the analysis by participants (1223 vs. $1248 \mathrm{~ms}), F_{1}(1,123)=4.94$, $F_{2}(1,30)=1.56$ (n.s.), and in structurally primed than unprimed sentences (1212 vs. $1259 \mathrm{~ms}), F_{1}(1,123)=21.28$, $F_{2}(1,30)=29.45$.

Overall, speakers also initiated sentences with related objects more slowly than sentences with unrelated objects $(1225$ vs. $1246 \mathrm{~ms}), F_{1}(1,123)=3.42, p=.067, F_{2}(1,30)=$ 6.60 , suggesting interference from retrieval of the second object name. This pattern was expected to vary across conditions with lexical and structural availability. On trials beginning with higher-frequency words, unprimed sentences with related and unrelated words had similar onsets (1242 and $1234 \mathrm{~ms}$ respectively), pointing to a narrow, subphrasal scope of planning. However, repetition of structure extended planning scope to include both words in the complex noun phrase (Fig. 2a): speakers initiated primed sentences with related nouns $51 \mathrm{~ms}$ later than sentences with unrelated nouns (1229 vs. $1178 \mathrm{~ms}$ ). Thus the structural priming effect was $56 \mathrm{~ms}$ in sentences with unrelated nouns and only $12 \mathrm{~ms}$ in sentences with related nouns, where the facilitatory effect of structural priming on speech onsets was offset by the presence of semantic interference. The 95\% confidence interval for these differences, calculated from the error term of the three-way interaction in the by-participant analysis of variance $\left(F_{1}(1,123)=2.66\right.$, $\left.p=.11, F_{2}(1,30)=6.10\right)$, was \pm 39 ms.

A different pattern was observed in sentences where the lower-frequency object was named first (Fig. 2b). Unprimed sentences with related and unrelated objects had similar onsets ( 1290 and 1259 ms respectively), which again suggests a subphrasal scope of planning in these conditions. Structural priming facilitated production of both types of sentences: onsets for primed sentences with related and unrelated words were shorter than in unprimed sentences (by 72 and $48 \mathrm{~ms}$, respectively) and very similar 

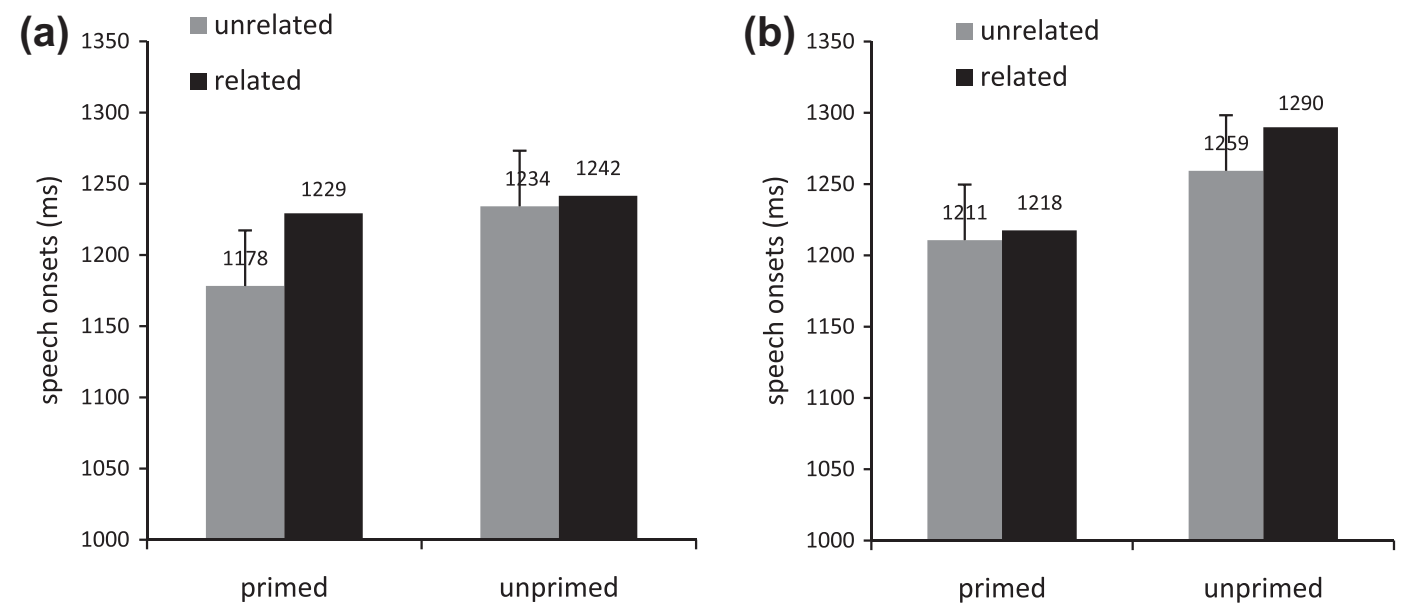

Fig. 2. Mean speech onsets in Experiment 1 for target sentences beginning with (a) high-frequency words and (b) low-frequency words.

to each other (1218 and $1211 \mathrm{~ms}$ respectively), indicating no interference from the second object name. So, despite the availability of a structure early in the formulation process, there was no observable change in planning scope in these conditions.

\section{Discussion}

The results confirm that the ease of lexical encoding and structural formulation have consequences for production at speech onset, but changes in planning scope were only observed with changes in structural accessibility. While speakers employed a subphrasal scope of planning in unprimed sentences, irrespective of the frequency of the first word, an observable expansion of planning scope occurred in primed sentences beginning with higher-frequency words. Repetition of structure reduced the overall costs of building a structural frame, replicating Smith and Wheeldon (2001), but priming was almost completely eliminated in sentences with related words, showing that speakers experienced interference from retrieval of the second object name before speech onset. This indicates a shift from a subphrasal to a phrasal scope of planning when structural frames were easier to generate.

Identifying the ease of structural formulation as a modulator of planning scope lends support to accounts favoring structurally incremental planning. On this view, a sentence frame is generated in the early stages of sentence formulation (Bock et al., 2003, 2004; Griffin \& Bock, 2000 ), and the easier this process was, the more extensive speakers' preparation of material following the sentenceinitial word appeared to be. When a frame took longer to generate, participants began speaking having constructed only a minimal part of the phrasal frame needed to begin their sentences. This makes narrow planning scope a natural consequence of slower or impeded structural processing, rather than a default for linguistic planning in such sentences.

As expected, there was no evidence of semantic interference at speech onset in primed sentences beginning with lower-frequency words. As an isolated finding, the lack of competition between target words in this condition would point to a narrow, subphrasal scope of planning even when generating a familiar phrasal structure. However, in the context of the effects obtained with sentences with higher-frequency words, lexical frequency is unlikely to be the only constraint, or even the strongest constraint, on planning scope: if it were, speakers would have adopted a phrasal planning scope in all sentences beginning with high-frequency words, structurally primed or not. The difference between sentences with higher- and lower-frequency words suggests that shifts towards phrasal planning when structural formulation was easier were likely only observable when the ease of retrieving the first word allowed simultaneous retrieval of the next word: i.e., the inaccessibility of the sentence-initial word obscured the benefits of generating a primed structure for sentence planning. On this account, a wider scope of planning should be observable even with lower-frequency words if they become easier to retrieve. This question was addressed in Experiment 2 with experimentally-induced changes in the accessibility of low-frequency words.

\section{Experiment 2}

The scope of linguistic planning in paradigms like the one employed here is normally evaluated by testing when speakers activate words. Since speech onsets are sensitive to variables like lexical frequency, it is not surprising that a lexical bottleneck in production should produce a bottleneck in planning, influencing the timing of word retrieval even in structurally primed sentences (as in Experiment 1). Experiment 2 tested the flexibility of this constraint by comparing changes in planning scope as a function of recent changes in lexical accessibility.

The ease of lexical retrieval in natural speech varies with experience, e.g., with changes in the salience and accessibility of lexical items across discourse topics, multiple mentions of the same referent, etc. The experimental 
analog of experience-dependent boosts in lexical accessibility is faster word retrieval following exposure or production of these lexical forms. Repetition priming effects are surprisingly robust and long-lasting (Wheeldon \& Monsell, 1992), and, in fact, a word's estimated overall frequency may be nothing more than a cumulative, long-term repetition effect (Zevin \& Seidenberg, 2002). If the processes responsible for grammatical encoding are sensitive to fine differences in the moment-to-moment availability of words as well as structures, then sentence planning should be influenced by long-term and short-term repetition effects alike: recent production of low-frequency words should have similar consequences for planning scope as production of high-frequency words. Thus speakers should show a shift from a subphrasal to a phrasal scope of planning in sentences where hard-to-retrieve words are repeated compared to sentences beginning with non-repeated words, replicating the effects shown in Experiment 1 with sentences beginning with high- and low-frequency words respectively.

So in Experiment 2, speakers completed two allegedly unrelated tasks. First they carried out a sentence completion task where they read sentences ending in an incompletely spelled but contextually unambiguous word. They were asked to complete this word with the first thing that came to mind. A subset of these sentences ended with lowfrequency words later used on target trials. In the second part of the experiment, participants completed a picturenaming task that was identical in most respects to Experiment 1: target displays elicited sentences with semantically related or unrelated words produced in a complex noun phrase. However, the low-frequency member of each semantic pair was always produced first, and half of the target sentences began with an object whose name had been produced earlier in the sentence completion task. With a decrease in retrieval costs for these words, speakers were expected to show evidence of phrasal planning in primed sentences.

\section{Method}

\section{Participants}

Ninety-six undergraduates from the University of Illinois participated in exchange for course credit. All were native speakers of English. Thirteen were replaced because they failed to produce a minimum of $60 \%$ scorable responses.

\section{Materials, procedure, and design}

The materials and procedure were adapted from Experiment 1 , with the addition of the sentence completion task. In this task participants read 42 sentences, of which 16 ended with a high-cloze probability, low-frequency word that would later appear on target trials in the picture-naming task (partially adapted from Griffin \& Bock, 1998). Of the remaining sentences, 20 ended with a word used later on filler trials, and six ended with words not used elsewhere in the experiment. Sentences were displayed on a computer screen one by one, and participants were instructed to read each sentence out loud and complete it with the first word that came to mind.
For the picture-naming task, participants were given the same instructions as in Experiment 1, and were assigned to one of 16 lists. Each item appeared in a different condition across lists. The design was again a three-factor within-participant and within-item factorial, crossing the factors of repetition of the sentence-initial word (repeated vs. non-repeated), semantic relatedness of the target words (related vs. unrelated), and structural priming (primed vs. unprimed). All analyses collapsed across the fourth factor (similarity in spatial layout of prime and target displays). Within lists, each participant saw four items in each cell of the design, and each item was seen by 12 participants in each cell of the design.

In each list, items were arranged so that no two items from the same condition were presented back to back. Prime-target pairs alternated between spatially and structurally primed and unprimed conditions. There were no more than two consecutive prime-target pairs with either semantically related or semantically unrelated words, and no more than two consecutive prime-target pairs beginning with a repeated or non-repeated word.

\section{Scoring and analyses}

The scoring was the same as in Experiment 1 and led to the exclusion of $23 \%$ of all trials. Trials in which participants paused between production of the first article and the first noun and in which speech onsets were longer than a condition-appropriate cutoff were also eliminated, leaving 2225 trials for analysis (72.4\% of all trials; see Table 3).

\section{Results}

As in Experiment 1, speakers initiated primed sentences earlier than unprimed sentences $(1127$ vs. $1167 \mathrm{~ms})$, $F_{1}(1,89)=8.86, F_{2}(1,30)=7.32$. Onsets were numerically shorter for sentences beginning with repeated words than sentences with non-repeated words (1136 vs. $1158 \mathrm{~ms}$ ), with a marginal main effect in the analysis by participants only, $F_{1}(1,89)=3.89, p=.052, F_{2}(1,30)=1.55$ (n.s.). Sentences with related words were also initiated more slowly than sentences with unrelated words (1166 vs. $1128 \mathrm{~ms}$ ), $F_{1}(1,89)=8.11, F_{2}(1,30)=12.12$. To evaluate the effects of lexical and structural availability on sentence planning across conditions, planned comparisons were made against a $95 \%$ confidence interval of $\pm 43 \mathrm{~ms}$, calculated from the error term of the three-way interaction in the by-participant analysis of variance $\left(F_{1}(1,89)=4.34, F_{2}(1,30)=1.89\right.$, n.s. $)$.

As expected, changes in the accessibility of the first word due to repetition priming were sufficient to produce a change in planning patterns (Fig. 3a). In sentences beginning with repeated words, unprimed sentences with related and unrelated words had similar onsets (1162 and $1155 \mathrm{~ms}$ respectively), consistent with a subphrasal scope of planning. In contrast, there was evidence of semantic interference in primed sentences, as speakers initiated primed utterances with related nouns $70 \mathrm{~ms}$ later than sentences with related nouns (1161 vs. $1091 \mathrm{~ms}$ ). Thus structural priming reduced onsets by $64 \mathrm{~ms}$ in sentences with unrelated nouns and by $1 \mathrm{~ms}$ in sentences with related nouns. In short, sentences beginning with repeated, 

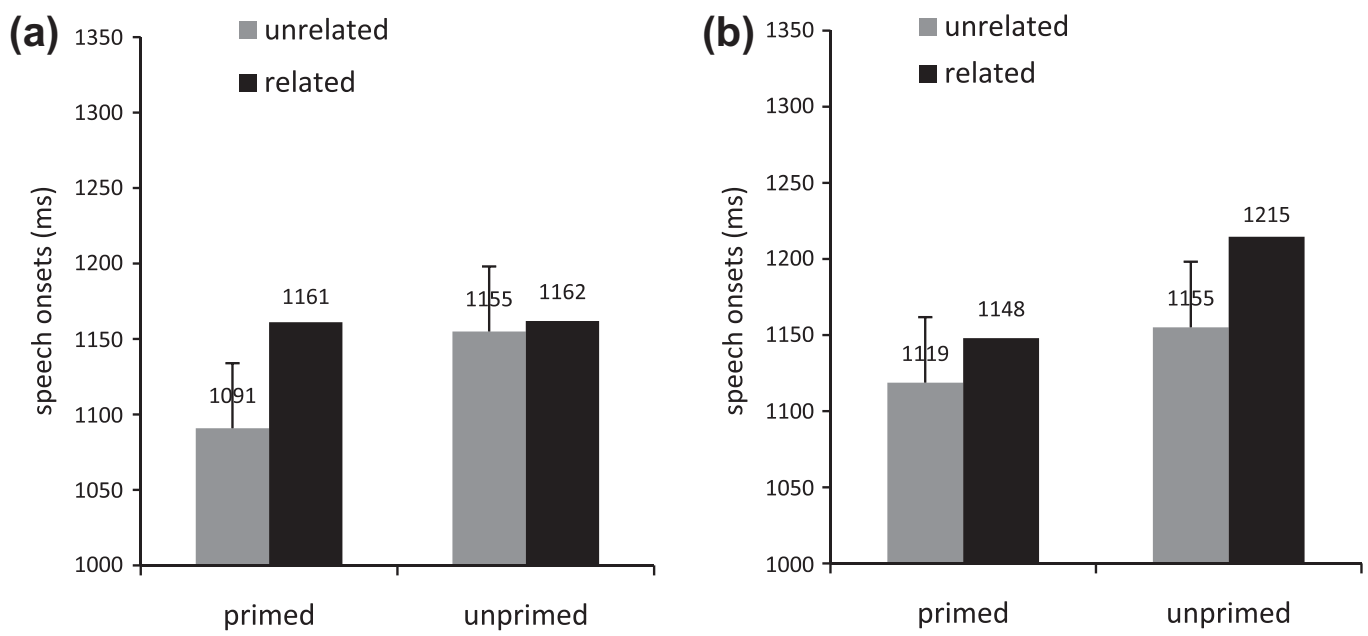

Fig. 3. Mean speech onsets in Experiment 2 for target sentences beginning with (a) repeated low-frequency words and (b) non-repeated low-frequency words.

low-frequency words behaved much like sentences with easily retrievable, high-frequency words in Experiment 1.

The results for primed sentences with less accessible words showed no comparable shift in planning scope (Fig. 3b): structural priming reduced speech onsets for sentences with related and unrelated nouns alike (by 67 and $36 \mathrm{~ms}$ respectively), suggesting that the width of the planning window did not change with repetition of structure. However, onsets in unprimed sentences with related nouns were $60 \mathrm{~ms}$ longer than in sentences with unrelated nouns (1215 vs. $1155 \mathrm{~ms}$ ), suggesting early retrieval of the second noun in these conditions. A similar trend was observed in Experiment 1, and possible explanations for these results are discussed below.

\section{Discussion}

Manipulations of structural and lexical accessibility in this study largely replicated the results of Experiment 1, and are consistent with structurally incremental planning. Lexical availability alone did not determine whether speakers employed a narrow or a wider planning window: rather, speakers appeared to engage in more extensive, phrasal planning when structural processing was easier to complete, and less extensive, subphrasal planning when they had not recently used the target phrasal structure. The within-item manipulation of lexical accessibility confirms that lexical properties of sentence-initial words determined only whether extended planning was observable or not: phrasal planning, as indexed by the presence of semantic interference, was, in fact, observed in sentences beginning with more accessible words only when these words were also produced in a repeated structure.

The only difference between the two experiments was in the speech onsets for unprimed sentences beginning with non-repeated, low-frequency words, suggesting the need for further explanations of how speakers approach the encoding of difficult information. More work is neces- sary to test the specific mechanisms responsible for these results, but, in theory, speakers have at least two options when planning sentence-initial material. The first option is to devote all resources to encoding the sentence-initial element before moving on to the rest of the sentence, which would result in a restricted scope of planning and thus comparable onsets in sentences with related and unrelated nouns (both when primed and unprimed) in this paradigm. The other possibility is that when encountering a low-frequency word, speakers may look ahead to search for contextual information that might facilitate retrieval of this word (see Wagner et al., 2010). This would produce semantic interference in sentences with related words, but looking ahead, in this case, may be a strategic decision rather than an outcome of phrasal planning proper.

The results for unprimed sentences beginning with low-frequency or non-repeated words support the second option: in Experiment 1, onsets for unprimed sentences with semantically related words were numerically longer than for sentences with unrelated words, and this difference reached significance in Experiment 2. Importantly, in both experiments, the results for primed sentences beginning with difficult-to-retrieve words showed no evidence of semantic interference, so it appears that speakers may consider the larger message context when encoding both words and structures is difficult.

Consistent with this explanation, a similar pattern was observed in a recent event-naming experiment where speakers described pictures of transitive events (Kuchinsky \& Bock, 2010). The pictures were either easy or hard to describe ("easy" pictures showed high-codability events described with a small set of verbs by most speakers, and "hard" pictures showed low-codability events described with a wider range of verbs) and involved characters that were also easy or hard to name. One of the characters was briefly cued with an attention-capture manipulation before picture onset, and the dependent variable was the proportion of sentences produced with the cued character 
in subject position. The results showed that when describing a difficult event in which the difficult-to-name characters had been cued, speakers were more likely to select the other character as subject, suggesting that they preferred to use other information in the event to begin their sentences when the cue directed their gaze to a difficult starting point. By analogy, in the current set of experiments, speakers may have looked ahead to the second object more often when they began a sentence with a less accessible, unprimed phrasal structure (i.e., when describing a difficult "event") and with a low-frequency word (i.e., a difficult-to-name object). In the current paradigm, such a strategy would result in semantic interference, and this finding may add an important qualification to theories of sentence planning based on manipulations of processing difficulty.

\section{General discussion}

\section{Lexical and structural effects on linguistic planning}

Earlier work on sentence planning showed considerable variability in planning scope for sentences consisting of simple and complex noun phrases, with speakers employing a clausal, phrasal, or a subphrasal planning scope in different studies (e.g., Griffin, 2001; Meyer, 1996, 1997; Smith \& Wheeldon, 1999). The current experiments examined whether variability in planning windows may be an outcome of the coordination of different production processes. The efficiency of grammatical encoding operations can change from sentence to sentence, as words can be easier or harder to retrieve and structures can be easier or harder to assemble, so changes in planning scope were assessed relative to fluctuations in formulation fluency: The structure of complex noun phrases in target sentences was either more or less familiar to speakers due to repetition of structure from the preceding prime trial, and lexical items were more or less familiar due to differences in cumulative frequency (Experiment 1) and recent usage (Experiment 2).

Most theories of language production take into account interactions between lexical retrieval and structure building, but the nature of these interactions remains a puzzle when the independent contributions of lexical and structural processes to sentence planning are difficult to identify (see Pickering \& Ferreira (2008), for a discussion of this problem in structural priming studies). The crucial comparison between lexical control and structural control of early sentence formulation in these experiments rests on the hypothesis that while both the processes of word retrieval and structure building can influence planning scope, they should do so in different ways.

Structural repetition and the ease of lexical retrieval reduced sentence onsets in both experiments, but only the manipulation affecting structural processing consistently accounted for observable shifts in planning scope. In sentences beginning with easily retrievable words, speakers experienced no semantic interference when using structures they had not recently produced: The structural plan is more difficult to generate or takes longer to generate in unprimed sentences, and participants appeared to initiate speech after encoding the smallest linguistic unit necessary to begin speaking (a definite article and the first object name). Retrieval of the second noun was a temporally distinct event in these sentences, possibly awaiting the completion of phonological encoding of the first noun (Meyer et al., 1998).

Fig. 4 summarizes these effects by showing the magnitude of semantic interference (or the difference between onsets in sentences with related and unrelated words) when an easily accessible word was produced first in the two experiments. A post hoc analysis carried out on pooled data from these experiments again showed the predicted main effect of structural priming, $F_{1}(1,212)=$ $7.08, F_{2}(1,60)=12.23$, and the magnitude of facilitation due to structural priming in sentences with unrelated words (56 and $64 \mathrm{~ms}$ in the two experiments respectively) was numerically comparable to that reported in earlier work (Smith \& Wheeldon, 2001). There was also a main effect of semantic relatedness of the target words, $F_{1}(1,212)=7.25, F_{2}(1,60)=10.61$, and an interaction between semantic relatedness and structural priming, $F_{1}(1,212)=4.57, F_{2}(1,60)=5.11$ (also see analysis (c) in Appendix B) confirming that semantic interference was only observed in one condition (i.e., in primed sentences).

This pattern of results is consistent with the main tenets of structural incrementality, or the view that a rudimentary structural frame is generated at the outset of sentence formulation without lexical support. If speakers begin to build a structure as soon as they have formulated at least part of the message they want to communicate, then the ease of generating this frame can have consequences for sentence planning: accordingly, speakers were able to use a phrasal planning window when a structural frame was repeated from trial to trial but not when structure was different. The results are difficult to reconcile with the assumptions of lexical incrementality, which posits that sentence planning is primarily lexically driven and thus that sentence structure "emerges" from the sequence of lexical retrieval operations. If word retrieval were a prerequisite for generating structures, the availability of

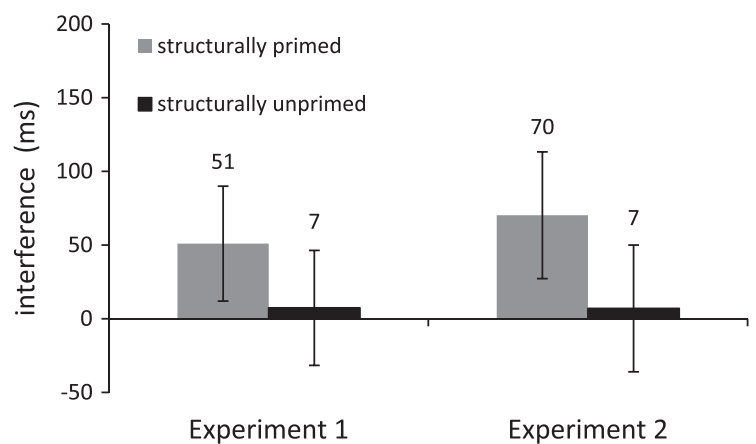

Fig. 4. Summary of semantic interference effects in sentences beginning with easily accessible words: high-frequency words in Experiment 1 and repeated, low-frequency words in Experiment 2. Bars show the difference between onsets in the neutral and semantic conditions for each experiment separately; the error bars are the 95\% confidence intervals for the difference between means obtained in each experiment. 
phrasal frames in structurally primed conditions should not have influenced planning patterns before retrieval of the first object name, and speakers should have used similarly-sized planning windows for the first sentence increment across conditions.

The results obtained with primed sentences beginning with less accessible words point to an important role of lexical variables in this process as well. Both experiments showed little evidence of semantic interference in these sentences: structural priming reduced onsets, $F_{1}(1,222)=$ 24.68, $F_{2}(1,60)=15.30$, but there was no interaction between structural priming and semantic relatedness, $F_{1}(1,222)=1.71, F_{2}(1,60)=2.44$ (also see analysis $(\mathrm{d})$ in Appendix B). Overall speakers did begin sentences with related words more slowly than sentences with unrelated words, $F_{1}(1,222)=6.86, F_{2}(1,60)=13.24$, suggesting some planning of the second noun before speech onset, but this difference was only apparent in the unprimed conditions rather than the primed conditions. The more important finding for present purposes is that when speakers had a structural frame that encouraged retrieval of two words in parallel (i.e., in the primed conditions), they adopted a narrow, subphrasal planning scope nevertheless.

As outlined earlier, at first glance this outcome may appear to be compatible with the claim that planning scope is primarily constrained by lexical factors, consistent with lexical incrementality. To distinguish between this possibility and the possibility of lexical factors modulating planning scope in conjunction with structural processes-a view consistent with structural incrementality-it is important to interpret this result in the context of the basic computational requirements of the linearization process in production (Bock, 1987). Speech onsets are often used to make inferences about the ease of retrieving words and generating structures (Corley \& Scheepers, 2002; Smith \& Wheeldon, 1999), but they are not an independent measure of structural processing: words must, of course, be produced one by one, whether speakers have begun building a multi-phrase structure and planning more than one word in parallel before speech onset or not. So, a lexical constraint in the execution of a sentence plan (or in the linearization of speech) need not automatically imply a constraint on its formulation as well: similarly, Wagner et al., 2010, propose that reductions in planning scope reflect the difficulty of filling slots in a syntactic structure rather than building the structure itself. To verify that lexical frequency is indeed a rate-limiting factor affecting the observability of extended planning when structures are easy to generate, Experiment 2 used a within-item manipulation of lexical accessibility to facilitate word retrieval and showed phrasal planning in structurally primed sentences beginning with these words.

The presence of semantic interference in unprimed sentences beginning with low-frequency words in Experiment 2 opens up the possibility that speakers approach the encoding of difficult material (less accessible words produced in a less "accessible" structural frame) in a different manner. Looking ahead to other elements in the to-bedescribed display may be a strategic choice motivated by the need to seek additional information to aid sentence construction. What repetition of structure can do in these cases is facilitate one aspect of the production process by reducing the costs of structure building, allowing speakers to begin the sentence earlier than they would be able to otherwise (i.e., showing a structural priming effect), but this hypothesis remains to be verified.

\section{Incrementality in language production}

These experiments demonstrate that questions about the breadth of planning scope can be restated as questions about the conditions under which planning may be more extensive or more limited. The coordination of lexical and structural processes within a flexible and efficient production system can produce systematically narrow or wider planning windows, but overall, the results of manipulations of encoding fluency favor structural incrementality over lexical incrementality: the size of sentence-initial sentence increments covaried with the ease of structural planning while the execution of this sentence plan depended on the accessibility of the words produced in these sentences, and not the other way around.

Priming complex noun phrases provides a strong test case for structurally incremental planning because such phrases are relatively uncomplicated structures with few internal dependencies. If the size of planning windows is a compromise between planning enough material to maintain fluency and planning as little as possible to avoid interference (Levelt \& Meyer, 2000; Martin et al., 2010), production of a noun phrase where word order is not contingent on verb selection or higher-level conceptual factors encourages lexically incremental planning (see Brown-Schmidt \& Konopka, 2008). This may be why evidence of subphrasal planning rather than phrasal or supra-phrasal planning is often obtained with such sentences (e.g., Griffin, 2001; Meyer, 1997) and why questions about more extensive sentence planning are predominantly addressed with more complex sentence types (Bock et al., 2003; Gleitman et al., 2007; Griffin \& Bock, 2000; Kuchinsky \& Bock, 2010). Facilitating structural processing in complex noun phrases may expand a planning window to the point of speakers activating a second noun early, but the speaker may nevertheless restrict planning to the sentence-initial noun because the identity of the second object has no consequences for their choice of sentence structure and may increase processing load (Dell, Oppenheim, \& Kittredge, 2008). Since encoding such phrases does not immediately lend itself to hierarchical planning, shifts towards phrasal planning are bound to be small; the fact that such shifts do occur suggests that structural incrementality is possible even in descriptions of simple spatial arrays. In theory, manipulating the ease of grammatical encoding in the production of more complex sentences should show comparable effects on the time course of sentence planning, although of course the range of relevant planning windows may be different.

Besides the possible match between the nature of the relationship between words in a complex noun phrase and the size of planning windows in these phrases, production also involves the strategic component of using planning windows that best suit the needs of the current communicative context. So, it is reasonable to assume that 

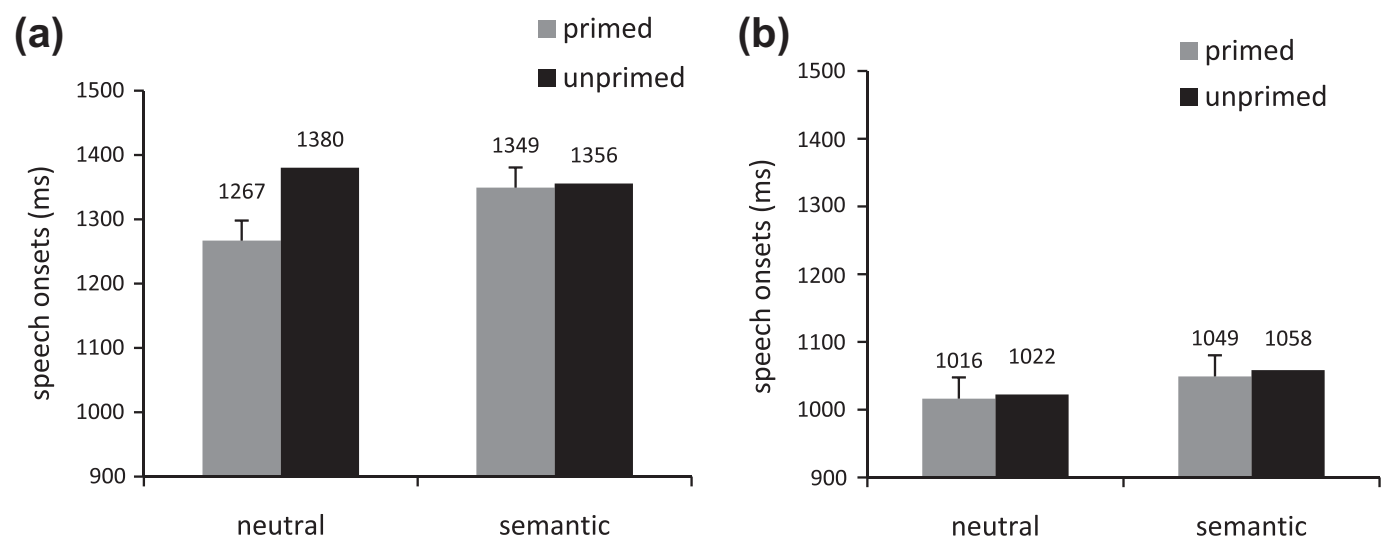

Fig. 5. Summary of results for (a) slow and (b) fast responders producing sentences beginning with easily accessible words in both experiments.

some speakers in these experiments may not have planned complex phrases as phrasal units but rather opted for planning their responses in smaller increments to maximize speed of communication (Levelt \& Meyer, 2000): this strategy should have reduced any effects of the lexical and structural manipulations. To verify the hypothesis that speed of responding is related to breadth of planning, post hoc analyses were carried out on data for sentences beginning with easily accessible words following a median split of participants by experiment into fast and slow responders (also see analysis (e) in Appendix B for a model including participants' mean onsets as a continuous covariate). An analysis of variance with the factors of semantic relatedness, structural priming, and speaker type showed a reliable three-way interaction, $F_{1}(1,212)=5.05$, $F_{2}(1,56)=6.35$, confirming that slow responders were the only ones who experienced interference in primed sentences with related words (Fig. 5a; also see Wagner et al. (2010), for similar results). They were also the only ones to begin structurally primed sentences more quickly than unprimed sentences, as shown by an interaction between structural repetition and speaker type, $F_{1}(1,212)=5.33$, $F_{2}(1,56)=3.64, p=.06$. Fast responders did not show reductions in speech onsets or changes in planning scope with increased availability of structure (Fig. 5b). The same pattern was observed in each experiment separately, so it is possible that the interaction between semantic relatedness and structural repetition was only reliable in the joint analysis due to considerable speaker variability in these datasets. ${ }^{3}$ Reasons for differences in production speed across speakers may include differences in working memory spans, allocation of attention, linguistic ability, etc., and causal links between these variables remain to be investigated.

\footnotetext{
3 The joint analysis for sentences beginning with less accessible words in the two experiments showed no interaction between semantic relatedness and structural priming, and this was true of both faster and slower responders. As in the analysis for sentences beginning with more accessible words, faster responders showed a much weaker structural priming effect than slow responders.
}

The implication for studies of linguistic planning is that multiple aspects of the production experience need to be taken into account to identify sources of variability in planning scope. Variability is likely both strategic and process-based: for example, speakers may want to allocate more resources or fewer resources to sentence planning as needed to meet their communicative goals, and variability in planning scope may then follow from processing constraints within that range of resources. Consequently, studies showing that linguistic planning is restricted to the first noun in a complex noun phrase and that the properties of this word account for all the variability in speech onsets need not automatically indicate a narrow planning scope, as speakers may exhibit a subphrasal scope of planning (Griffin, 2001; Meyer et al., 1998) for reasons other than reliance on a lexically incremental planning strategy. Regarding structural processing in particular, planning windows may be narrow when structures are repeated from trial to trial throughout an experiment (e.g., Griffin, 2001) and when speakers respond very quickly (e.g., as seen in the current experiments with slower responders), or may be small when structures take longer to generate: critically, the reasons for speakers employing a narrow scope of planning in these three cases are different.

One important question for models of sentence production is whether process-based variability in planning scope might occur within a range that maps onto grammatically relevant units. Strategically-motivated changes in planning scope may not speak to this question directly (e.g., see Ferreira \& Swets, 2002), but if variability can be reliably predicted from properties of the production system and from speaker characteristics, the upper bounds of planning windows may emerge from interactions between these factors. Mapping out the range of these windows may provide convergent evidence with studies estimating the size of "default" planning windows with different manipulations, so identifying bottlenecks in planning may be a complementary approach to one where units of planning are identified first and inferences about processes are made later. The evidence accumulated so far suggests the limits of planning windows for noun phrases lie roughly at phrasal 
boundaries (e.g., Allum \& Wheeldon, 2009; Martin et al., 2010; Wagner et al., 2010).

The specific relationship between bottlenecks in planning and architectural constraints remains to be determined, but in principle structural incrementality is compatible with production models where the processes responsible for word order are separate from lexical semantics (Chang et al., 2006), and is consistent with recent experimental work supporting the notion of structural planning preceding lexical access (Allum \& Wheeldon, 2009; Wagner et al., 2010). An obvious example of the benefits of this architecture is that relying on a sequencing system to control word order supports efficient planning and production: activating words as a structural frame calls for them ensures that words are inserted in the correct slots while minimizing processing load and the possibility of interference between, for example, words to be produced in different phrases (see Dell et al. (2008), for a review). Identifying potential bottlenecks in the formulation process provides one way of finetuning production models to explain variability within a clear set of architectural constraints.

\section{Structure in messages and structure in language}

A closely related question to the assessment of linguistic scope concerns the coordination of message planning and linguistic planning, and its contribution to variability in linguistic planning scope (Paul, 1880; Wundt, 1900). The Chang et al. (2006) model, for example, depends heavily on interactions between the conceptual system and the sequencing system. Message planning involves conceptualizing relationships between the things that the speaker wants to say something about, so, all else being equal, structural and lexical effects of the type observed in these experiments may also depend on the complexity and completeness of the message plan. One of the critical questions in such studies often regards the levels-conceptual, linguistic, or both-at which advance preparation occurs: by default, when message preparation is limited, speakers can only prepare smaller chunks of linguistic material, but when message planning is easier and more information can be passed onto linguistic encoding processes, there is room for variability in the output of these processes. Thus, systematic changes in planning scope may only occur when speakers can first engage in extensive conceptual planning.

To control for possible differences in the outcome of linguistic encoding due to the complexity of conceptual processing, both experiments included a manipulation of spatial similarity between prime and target displays. The high degree of isomorphism between spatial structure and sentence structure in these experiments makes it difficult to dissociate visual and linguistic factors, but the ease of message encoding can be manipulated across trials much like the ease of structural and lexical encoding. Thus half the time, speakers were cued to begin prime and target sentences with an object appearing in either the top or bottom row of the display, and half the time, the cued location of the first object on prime and target trials was different (see Fig. 1). Since speakers were asked to describe the spatial relationships between objects in a display, this manipulation was expected to increase or decrease the ease of planning these relationships. Statistical analyses for each experiment did not include this factor due to a high number of empty cells in the multi-factor designs; however, pooling the data from both experiments and comparing the results of fast and slow responders showed an important role of speaker differences in message planning as well. Three-factor analyses were carried out on data from fast and slow responders separately (and only for sentences beginning with easily accessible words), comparing the effects of semantic relatedness, structural repetition, and similarity in spatial layout on sentence planning (analysis (f) in Appendix B with mean onsets as a covariate combines data from fast and slow responders).

For slower responders, structural priming expanded the scope of planning in targets beginning with easily retrievable nouns irrespective of the spatial similarity in prime and target displays. The analysis performed on this subset of participants showed an interaction between semantic relatedness and structural priming, $F_{1}(1,50)=6.44$, $F_{2}(1,41)=4.91$, but no interactions with spatial similarity all $F$ 's $<1$. The only reliable effect of spatial similarity was to reduce speech onsets on trials with similar displays compared to trials with dissimilar displays (a main effect with $\left.F_{1}(1,50)=8.69, F_{2}(1,41)=5.79\right)$. The difference in the magnitude of structural priming between sentences with related and unrelated words was numerically larger when there was spatial overlap between prime and target displays, confirming that the ease of mapping perceptual information onto a linguistic expression can facilitate linguistic planning (Bock et al., 2003).

Unlike the slower responders, participants who initiated speech more quickly showed no structural priming, $F_{1}(1,61)=.001, F_{2}(1,39)=.34$. Spatial similarity between primes and targets again reduced onset latencies overall, $F_{1}(1,61)=7.72, F_{2}(1,39)=5.20$, but did not interact with other factors, all $F$ s $<2$, suggesting that spatial properties of the prime and target displays did not modulate planning scope in this group of speakers. Formulation of a nonminimal message chunk is a prerequisite for observing any variability in linguistic planning, but these results suggest that the degree to which speakers plan ahead may be better predicted by taking individual participant characteristics into account than message complexity alone.

\section{Conclusions}

Questions regarding planning scope in extemporaneous speech can be addressed by considering both the products and the processes of speech production. In principle, planning windows need only be large enough to encode the smallest meaningful message unit, such as the identity of a single object, but speakers may plan their utterances more or less extensively depending on their communicative goals and on their linguistic experience. The current experiments tested the latter hypothesis, by evaluating whether relaxing lexical and structural constraints on sentence building can change planning scope within complex noun phrases. Work examining production of longer sentences supports accounts favoring structurally incremental planning, and the predictions of structural incrementality were borne out for simpler sentences in these experiments 
as well: when structure-relevant information was readily available, speakers showed a shift towards phrasal planning by encoding more than the sentence-initial element before speech onset.

The ease of linguistic encoding may be only one among several factors influencing the breadth of planning scope. A flexible production system must allow for interactions between lexical and structural processes during grammatical encoding as well as interactions between message-level information and grammatical encoding, so examining variability in planning windows for different sentence types may show qualitatively and quantitatively different changes in planning scope than the effects obtained with complex noun phrases. The width of planning windows may depend, for example, on the size of the units most relevant for expressing a specific relationship between objects or events in a message. More generally, the current work suggests that changes in planning scope should be predictable from the larger production context, adding an important qualification to the development of a unified theory of linguistic planning. The results also show a contribution of speaker-specific variables to changes in planning scope: Additional information about speaker characteristics is necessary to identify causes of these individual differences and thus weigh the contributions of this factor to variability in planning scope.

\section{AcknowledgementsAcknowledgments}

The experiments reported in this paper were carried out at the University of Illinois at Urbana-Champaign as part of the author's doctoral research. I'd like to extend many thanks to Kay Bock, Antje Meyer, Sarah Brown-Schmidt, Gary Dell, Aaron Benjamin, Cindy Fisher, and Duane Watson for helpful comments and discussions, Scott Fraundorf and Dan Dediu for assistance with statistical analyses, as well as Matt Rambert, Javier Ospina, and numerous research assistants for assistance with data collection.

\section{Appendix A}

Semantically related target words used in Experiments 1 and 2 (the higher-frequency word is listed first). map, globe

bed, couch

watch, clock

pie, cake

car, truck

brush, comb

duck, swan

plate, bowl

deer, moose

axe, saw

bee, fly

hat, cap

eye, ear

lion, tiger

coat, shirt

cow, pig

\author{
carrot, lettuce \\ fork, spoon \\ suitcase, backpack \\ eagle, parrot \\ necklace, earring \\ moon, star \\ tie, scarf \\ apple, cherry \\ chair, stool \\ toe, thumb \\ glove, sock \\ arm, leg \\ fish, whale \\ dress, pants \\ lips, nose \\ bottle, pitcher
}

\section{Appendix B}

All factors were coded using mean-centered contrast coding. Models (a) and (b) include a three-way interaction, following Jaeger (2009).

(a) The model fit to the data in Experiment 1 included a three-way interaction between semantic relatedness, structural priming, and lexical frequency. The interaction improved model fit as compared against a model with the same parameters but no three-way interaction, $\chi^{2}(1)=4.13$. Model fit was also improved by including random slopes by items for semantic relatedness and lexical frequency, and marginally improved by including random slopes by participants for structural priming.

(b) The model fit to the data in Experiment 2 included a three-way interaction between semantic relatedness, structural priming, and word repetition (i.e., lexical frequency). The interaction improved model fit as compared against a model with the same parameters but no three-way interaction, $\chi^{2}(1)=2.76, p<.10$. Model fit was also improved by including random slopes by participants for structural priming, and random slopes by items for semantic relatedness.

\begin{tabular}{|c|c|c|c|c|c|c|}
\hline \multirow[b]{2}{*}{ Predictor } & \multicolumn{3}{|c|}{ (a) Experiment 1} & \multicolumn{3}{|c|}{ (b) Experiment 2} \\
\hline & Estimate & $S E$ & $t$ & Estimate & $S E$ & $t$ \\
\hline Intercept & 1247.29 & 22.81 & $54.68^{*}$ & 1168.57 & 25.00 & $46.75^{*}$ \\
\hline Semantic relatedness & 41.83 & 14.17 & $2.95^{*}$ & 52.81 & 14.61 & $3.61^{*}$ \\
\hline Structural priming & -49.98 & 9.41 & $-5.31^{*}$ & -42.23 & 10.37 & $-4.07^{*}$ \\
\hline Lexical frequency & 24.81 & 19.14 & 1.30 & 20.25 & 9.46 & $2.14^{*}$ \\
\hline Interaction: Semantic relatedness $\times$ Structural priming & 3.56 & 18.09 & .20 & 13.29 & 18.92 & .70 \\
\hline Interaction: Semantic relatedness $\times$ Lexical frequency & -9.64 & 18.29 & -.53 & 12.14 & 18.92 & .64 \\
\hline Interaction: Structural priming $\times$ Lexical frequency & -19.81 & 18.11 & -1.09 & -7.50 & 18.90 & -.40 \\
\hline $\begin{array}{l}\text { Interaction: Semantic relatedness } \times \text { Structural } \\
\text { priming } \times \text { Lexical frequency }\end{array}$ & -73.48 & 36.13 & $-2.03^{*}$ & -62.80 & 37.83 & $-1.66^{\dagger}$ \\
\hline
\end{tabular}


(c) The first model combining data from the two experiments was fit to speech onsets for sentences beginning with easily accessible words. The model included an interaction between semantic relatedness and structural priming, random slopes by participants for structural priming, and random slopes for items for semantic relatedness. The two-way interaction improved model fit, as compared against a model with the same parameters but no interaction, $\chi^{2}(1)=5.38$.

(d) The second model combining data from the two experiments was fit to speech onsets for sentences easily accessible words using participants' mean onsets (response speed) as a covariate. The model included an interaction between semantic relatedness and structural priming, and random slopes for items for semantic relatedness and response speed. The inclusion of mean speech onsets as a continuous predictor interacting with both fixed factors improved model fit, as compared against a model with the same parameters but no three-way interaction, $\chi^{2}(1)=5.05$. This model was also a better fit to the data than model (c) which did not include response speed as a covariate, $\chi^{2}(5)=577.4$.

\begin{tabular}{llll}
\hline Predictor & Estimate & $S E$ & $t$ \\
\hline Intercept & 1194.61 & 13.34 & $89.56^{*}$ \\
Semantic relatedness & 41.68 & 10.55 & $3.95^{*}$ \\
Structural priming & -36.96 & 8.99 & $-4.11^{*}$ \\
Response speed & 1.05 & .03 & $35.22^{*}$ \\
Interaction: Semantic relatedness $\times$ Structural priming & 43.14 & 17.96 & $2.40^{*}$ \\
Interaction: Semantic relatedness $\times$ Response speed & .03 & .05 & .57 \\
Interaction: Structural priming $\times$ Response speed & -.13 & .05 & $-2.44^{*}$ \\
Interaction: Semantic relatedness $\times$ Structural priming $\times$ Response speed & .23 & .10 & $2.25^{*}$ \\
\hline
\end{tabular}

$p<.05$.

beginning with less accessible words. The model included two additive fixed factors (semantic relatedness and structural priming), random slopes by participants for structural priming, and random slopes for items for semantic relatedness. Adding a two-way interaction between semantic relatedness and structural priming did not improve model fit, $\chi^{2}(1)=1.85, p=.17$. (f) Comparing results for fast and slow responders on trials with and without spatial similarity between primes and targets, a model combining data from the two experiments was fit to data for sentences beginning with easily accessible words. The model had the same parameters as model (e) above, with the addition of spatial similarity as a fixed factor. The inclusion of this factor as an additive effect improved model fit relative to model (e), $\chi^{2}(1)=22.51$.

\begin{tabular}{|c|c|c|c|c|c|c|}
\hline \multirow[b]{2}{*}{ Predictor } & \multicolumn{3}{|c|}{ (c) Accessible words } & \multicolumn{3}{|c|}{ (d) Less accessible words } \\
\hline & Estimate & SE & $t$ & Estimate & SE & $t$ \\
\hline Intercept & 1197.12 & 18.00 & $66.50^{*}$ & 1222.33 & 17.98 & $68.00^{*}$ \\
\hline Semantic relatedness & 41.92 & 11.01 & $3.81^{*}$ & 47.13 & 12.85 & $3.67^{*}$ \\
\hline Structural priming & -40.34 & 9.97 & $-4.05^{*}$ & -52.83 & 9.92 & $-5.33^{*}$ \\
\hline Interaction: Semantic relatedness $\times$ Structural priming & 43.07 & 18.55 & $2.32^{*}$ & - & - & - \\
\hline
\end{tabular}

(e) Comparing results for fast and slow responders, a model combining data from the two experiments was fit to speech onsets for sentences beginning with

\section{References}

Allum, P. H., \& Wheeldon, L. R. (2007). Planning scope in spoken sentence production: The role of grammatical units. Journal of Experimental Psychology: Learning, Memory, and Cognition, 33, 791-810.

Allum, P. H., \& Wheeldon, L. R. (2009). Scope of lexical access in spoken sentence production: Implications for the conceptual-syntactic interface. Journal of Experimental Psychology: Learning, Memory, and Cognition, 35, 1240-1255.

Ayora, P., Janssen, N., Dell'Acqua, R., \& Alario, F. X. (2009). Attentional requirements for the selection of words from different grammatical categories. Journal of Experimental Psychology: Learning, Memory and Cognition, 35, 1344-1351.

Baayen, R. H., Davidson, D. J., \& Bates, D. M. (2008). Mixed-effects modeling with crossed random effects for subjects and items. Journal of Memory and Language, 59, 390-412.

Bates, E., \& MacWhinney, B. (1982). Functionalist approaches to grammar. In E. Wanner \& L. R. Gleitman (Eds.), Language acquisition: The state of the art (pp. 173-218). Cambridge, England: Cambridge University Press.

Bock, J. K. (1982). Toward a cognitive psychology of syntax: Information processing contributions to sentence formulation. Psychological Review, 89, 1-47.

Bock, J. K. (1986). Syntactic persistence in language production. Cognitive Psychology, 18, 355-387.

Bock, J. K. (1987). Co-ordinating words and syntax in speech plans. In A. W. Ellis (Ed.), Progress in the psychology of language (pp. 337-390). London: Erlbaum. 
Bock, J. K. (1990). Structure in language: Creating form in talk. American Psychologist, 45, 1221-1236.

Bock, J. K., \& Griffin, Z. M. (2000). The persistence of structural priming: Transient activation or implicit learning? Journal of Experiment Psychology: General, 129, 177-192.

Bock, J. K., Irwin, D. E., Davidon, D. J., \& Levelt, W. J. M. (2003). Minding the clock. Journal of Memory and Language, 48, 653-685.

Bock, J. K., Irwin, D. E., \& Davidson, D. J. (2004). Putting first things first. In J. M. Henderson \& F. Ferreira (Eds.), The interface of language, vision, and action: Eye movements and the visual world (pp. 249-278). New York, NY: Psychology Press.

Bock, K., \& Levelt, J. W. M. (1994). Language production: Grammatical encoding. In M. A. Gernsbacher (Ed.), Handbook of psycholinguistics (pp. 945-984). Orlando, FL: Academic Press.

Bock, J. K., \& Warren, R. K. (1985). Conceptual accessibility and syntactic structure in sentence formulation. Cognition, 21, 47-67.

Bonin, P., Malardier, N., Meot, A., \& Fayol, M. (2006). The scope of advance planning in written picture naming. Language and Cognitive Processes, 21, 205-237.

Branigan, H. P., Pickering, M. J., \& Cleland, A. A. (1999). Syntactic priming in written production: Evidence for rapid decay. Psychonomic Bulletin E Review, 6, 635-640.

Branigan, H., Pickering, M., \& Cleland, A. (2000). Syntactic co-ordination in dialogue. Cognition, 75, B13-B25.

Brown-Schmidt, S., \& Konopka, A. E. (2008). Little houses and casas pequeñas: Message formulation and syntactic form in unscripted speech with speakers of English and Spanish. Cognition, 109, 274-280.

Brown-Schmidt, S., \& Tanenhaus, M. K. (2006). Watching the eyes when talking about size: An investigation of message formulation and utterance planning. Journal of Memory and Language, 54, 592-609.

Chang, F., Dell, G. S., \& Bock, J. K. (2006). Becoming syntactic. Psychological Review, 113, 234-272.

Christianson, K., \& Ferreira, F. (2005). Conceptual accessibility and sentence production in a free word order language (Odawa). Cognition, 98, 105-135.

Clark, H. H. (2002). Speaking in time. Speech Communication, 36, 5-13.

Clark, H. H., \& Clark, E. V. (1977). Psychology and language: An introduction to psycholinguistics. New York: Harcourt Brace Jovanovich.

Clark, H. H., \& Wasow, T. (1998). Repeating words in spontaneous speech. Cognitive Psychology, 37, 201-242.

Cleland, A. A., \& Pickering, M. J. (2003). The use of lexical and syntactic information in language production: Evidence from the priming of noun-phrase structure. Journal of Memory and Language, 49, 214-230.

Cohen, J. D., MacWhinney, B., Flatt, M., \& Provost, J. (1993). PsyScope: An interactive graphic system for designing and controlling experiments in the psychology laboratory using Macintosh computers. Behavior Research Methods, Instruments, \& Computers, 25, 257-271.

Cooper, W. E., \& Ross, J. R. (1975). World order. In R. E. Gleitman, L. J. San, \& T. J. Vance (Eds.), Papers from the parasession on functionalism (pp. 63-111). Chicago: Chicago Linguistic Society.

Corley, M., \& Scheepers, C. (2002). Syntactic priming in English sentence production: Categorical and latency evidence from an Internet-based study. Psychonomic Bulletin \& Review, 9, 126-131.

Dell, G. S. (1986). A spreading-activation theory of retrieval in sentence production. Psychological Review, 93, 283-321.

Dell, G. S., Burger, L. K., \& Svec, W. R. (1997). Language production and serial order: A functional analysis and a model. Psychological Review, 104, 123-147.

Dell, G. S., Oppenheim, G. M., \& Kittredge, A. K. (2008). Saying the right word at the right time: Syntagmatic and paradigmatic interference in sentence production. Language and Cognitive Processes, 23, 583-608.

Ferreira, V. S. (1996). Is it better to give than to donate? Syntactic flexibility in language production. Journal of Memory and Language, 35 , 724-755.

Ferreira, V. S., \& Bock, J. K. (2006). The functions of structural priming. Language and Cognitive Processes, 21, 1011-1029.

Ferreira, V. S., \& Dell, G. S. (2000). Effect of ambiguity and lexical availability on syntactic and lexical production. Cognitive Psychology, $40,296-340$

Ferreira, V. S., \& Pashler, H. (2002). Central bottleneck influences on the processing stages of word production. Journal of Experimental Psychology: Learning, Memory, and Cognition, 28, 1187-1199.

Ferreira, F., \& Swets, B. (2002). How incremental is language production? Evidence from the production of utterances requiring the computation of arithmetic sums. Journal of Memory and Language, $46,57-84$.
Ferreira, F., \& Swets, B. (2005). The production and comprehension of presumptive pronouns in relative clause "island" contexts. In Cutler (Ed.), Twenty-first century psycholinguistics: Four cornerstones (pp. 263-278). Mahwah, New Jersey: Lawrence Erlbaum Associates.

Fisher, C. (2002). The role of abstract syntactic knowledge in language acquisition: A reply to Tomasello (2000). Cognition, 82, 259-278.

Fraundorf, S., \& Watson, D. G. (2008). Dimensions of variation in disfluency production in discourse. In J. Ginzburg, P. Healey, \& Y. Sato (Eds.), Proceedings of LONDIAL 2008, the 12th Workshop on the Semantics and Pragmatics of Dialogue (pp. 131-138). London: King's College London.

Garnsey, S. M., Pearlmutter, N. J., Myers, E., \& Lotocky, M. A. (1997). The contributions of verb bias and plausibility to the comprehension of temporarily ambiguous sentences. Journal of Memory and Language, $37,58-93$.

Gleitman, L. R., January, D., Nappa, R., \& Trueswell, J. C. (2007). On the give and take between event apprehension and utterance formulation. Journal of Memory and Language, 57, 544-569.

Gries, S. T. (2005). Syntactic priming: A corpus-based approach. Journal of Psycholinguistic Research, 34, 365-399.

Griffin, Z. M. (2001). Gaze durations during speech reflect word selection and phonological encoding. Cognition, 82, B1-B14

Griffin, Z. M. (2003). A reversed length effect in coordinating the preparation and articulation of words in speaking. Psychonomic Bulletin \& Review, 10, 603-609.

Griffin, Z. M., \& Bock, J. K. (1998). Constraint, word frequency, and the relationship between lexical processing levels in spoken word production. Journal of Memory and Language, 38, 313-338.

Griffin, Z. M., \& Bock, J. K. (2000). What the eyes say about speaking. Psychological Science, 11, 274-279.

Griffin, Z. M., \& Spieler, D. H. (2006). Observing the what and when of language production for different age groups by monitoring speakers' eye movements. Brain and Language, 99, 272-288.

Hartsuiker, R. J., Bernolet, S, Schoonbaert, S, Speybroeck, S. \& Vanderelst, D. (2008). Syntactic priming persists while the lexical boost decays: Evidence from written and spoken dialogue. Journal of Memory and Language, 58, 214-238.

Hartsuiker, R. J., Pickering, M. J., \& Veltkamp, E. (2004). Is syntax separate or shared between languages? Cross-linguistic syntactic priming in Spanish-English bilinguals. Psychological Science, 15, 409-414.

Hartsuiker, R. J., \& Westenberg, C. (2000). Word order priming in written and spoken sentence production. Cognition, 75, B27-B39.

Jaeger, F. (2009). Lmer interactions in factorial designs. Message posted to RLang mailing list. <http://pidgin.ucsd.edu/pipermail/r-lang/2009July/000182.html> (July 31).

Kaschak, M. P., \& Borregine, K. L. (2008). Is long-term structural priming affected by patterns of experience with individual verbs? Journal of Memory and Language, 58, 862-878.

Kempen, G., \& Hoenkamp, E. (1987). An incremental procedural grammar for sentence formulation. Cognitive Science, 11, 201-258.

Konopka, A. E., \& Bock, K. (2009). Lexical or syntactic control of sentence formulation? Structural generalizations from idiom production. Cognitive Psychology, 58, 68-101.

Kucera, W. N., \& Francis, H. (1982). Frequency analysis of English usage: Lexicon and grammar. Boston, MA: Houghton Mifflin.

Kuchinsky, S. E., \& Bock, K. (2010). From seeing to saying: Perceiving, planning, producing. Paper presented at the 23rd meeting of the CUNY Conference on Human Sentence Processing, New York (March).

Kuchinsky, S. E. (2010). From seeing to saying: Perceiving, planning, producing. Unpublished doctoral dissertation. University of Illinois at Urbana-Champaign.

Kuiper, K. (1996). Smooth talkers: The linguistic performance of auctioneers and sportscasters. Mahwah, New Jersey: Lawrence Erlbaum Associates.

Levelt, W. J. M. (1989). Speaking: From intention to articulation. Cambridge, MA: MIT Press.

Levelt, W. J. M., \& Meyer, A. S. (2000). Word for word: Multiple lexical access in speech production. European Journal of Cognitive Psychology, $12,433-452$.

Lindsley, J. R. (1975). Producing simple utterances: How far ahead do we plan? Cognitive Psychology, 7, 1-19.

Loebell, H., \& Bock, J. K. (2003). Structural priming across languages. Linguistics, 41, 791-824.

Malpass, D., \& Meyer, A. S. (2010). The time course of name retrieval during multiple-object naming: Evidence from extrafoveal-on-foveal objects. Journal of Experimental Psychology: Learning, Memory, and Cognition, 36, 523-537. 
Martin, R. C., Crowther, J. E., Knight, M., Tamborello, F. P., \& Yang, C. (2010). Planning in sentence production: Evidence for the phrase as a default planning scope. Cognition, 116, 177-192.

Meyer, A. S. (1996). Lexical access in phrase and sentence production: Results from picture-word interference experiments. Journal of Memory \& Language, 35, 477-496.

Meyer, A. S. (1997). Conceptual influences on grammatical planning units. Language and Cognitive Processes, 12, 859-863.

Meyer, A. S., \& Lethaus, F. (2004). The use of eye tracking in studies of sentence generation. In J. M. Henderson \& F. Ferreira (Eds.), The interface of language, vision, and action: Eye movements and the visual world (pp. 191-212). New York: Psychology Press.

Meyer, A. S., Ouellet, M., \& Häcker, C. (2008). Parallel processing of objects in a naming task. Journal of Experimental Psychology: Learning, Memory, and Cognition, 34, 982-987.

Meyer, A. S., Roelofs, A., \& Levelt, W. J. M. (2003). Word length effects in object naming: The role of a response criterion. Journal of Memory and Language, 48, 131-147.

Meyer, A. S., Sleiderink, A. M., \& Levelt, W. J. M. (1998). Viewing and naming objects: Eye movements during noun phrase production. Cognition, 66, B25-B33.

Morgan, J. L., \& Meyer, A. S. (2005). Processing of extrafoveal objects during multiple-object naming. Journal of Experimental psychology: Learning, Memory, and Cognition, 31, 428-442.

Paul, H. (1880). Prinzipien der Sprachgeschichte [Principles of the history of language]. Leipzig: Niemeyer.

Pickering, M. J., \& Branigan, H. (1998). The representation of verbs: Evidence from syntactic priming in language production. Journal of Memory and Language, 39, 633-651.

Pickering, M. J., \& Ferreira, V. S. (2008). Structural priming: A critical review. Psychological Bulletin, 134, 427-459.

Potter, M. C., \& Lombardi, L. (1998). Syntactic priming in immediate recall of sentences. Journal of Memory and Language, 38, 265-282.

Prat-Sala, M., \& Branigan, H. P. (2000). Discourse constraints on syntactic processing in language production: A cross-linguistic study in English and Spanish. Journal of Memory and Language, 42, 168-182.

Roelofs, A. (2008). Attention, gaze shifting, and dual-task interference from phonological encoding in spoken word planning. Journal of Experimental Psychology: Human Perception and Performance, 34, 1580-1598.

Rosenthal, R., \& Rosnow, R. L. (1985). Contrast analysis: Focused comparisons in the analysis of variance. Cambridge, NY: Cambridge University Press.
Scheepers, C. (2003). Syntactic priming of relative clause attachments: Persistence of structural configuration in sentence production. Cognition, 89, 179-205.

Schriefers, H., \& Teruel, E. (1999). Phonological facilitation in the production of two-word utterances. European Journal of Cognitive Psychology, 11, 17-50.

Smith, M., \& Wheeldon, L. (1999). High level processing scope in spoken sentence production. Cognition, 73, 205-246.

Smith, M., \& Wheeldon, L. (2001). Syntactic priming in spoken sentence production - An online study. Cognition, 78, 123-164.

Smith, M., \& Wheeldon, L. (2004). Horizontal information flow in spoken sentence production. Journal of Experimental Psychology: Learning, Memory, and Cognition, 30, 675-686.

Snodgrass, J. G., \& Vaderwart, M. (1980). A standardized set of 260 pictures: Norms for name agreement, familiarity and visual complexity. Journal of Experimental Psychology: Human Learning $\mathcal{E}$ Memory, 6, 174-215.

Swets, B., Jacovina, M. E., \& Gerrig, R. J. (2008). Individual differences in the planning scope of language production. Paper presented at the 49st meeting of the Psychonomic Society, Chicago, IL (November).

Szekely, A., Jacobsen, Th., D'Amico, S., Devescovi, A., Andonova, E., Herron, D., et al. (2004). A new on-line resource for psycholinguistic studies. Journal of Memory and Language, 51, 247-250.

Szmrecsanyi, B. (2004). Persistence phenomena in the grammar of spoken English. Unpublished doctoral dissertation, Albert-LudwigsUniversität Freiburg, Freiburg, Germany.

Tomasello, M. (2000). Do young children have adult syntactic competence? Cognition, 74, 209-253.

Van Der Meulen, F. F., Meyer, A. S., \& Levelt, W. J. M. (2001). Eye movements during the production of nouns and pronouns. Memory $\mathcal{E}^{\circ}$ Cognition, 29, 512-521.

Wagner, V., Jescheniak, J. D., \& Schriefers, H. (2010). On the flexibility of grammatical advance planning during sentence production: Effects of cognitive load on multiple lexical access. Journal of Experiment Psychology: Learning, Memory, and Cognition, 36, 423-440.

Wheeldon, L. R., \& Monsell, S. (1992). The locus of repetition priming of spoken word production. Quarterly Journal of Experimental Psychology, 44, 723-761.

Wundt, W. (1900). Völkerpsychologie: EineUntersuchung der Entwicklungsgesetze von Sprache, Mythus und Sitte (Vol. 1). Die Sprache [Language]. Leipzig: Kroner-Engelmann.

Zevin, J. D., \& Seidenberg, M. S. (2002). Age-of-acquisition effects in reading and other tasks. Journal of Memory and Language, 47, 1-29. 